\title{
Discontinuous Hamiltonian Finite Element Method for Linear Hyperbolic Systems
}

\author{
Yan Xu · Jaap J.W. van der Vegt • Onno Bokhove
}

Received: 19 October 2007 / Revised: 24 January 2008 / Accepted: 30 January 2008 /

Published online: 1 March 2008

(c) The Author(s) 2008. This article is published with open access at Springerlink.com

\begin{abstract}
We develop a Hamiltonian discontinuous finite element discretization of a generalized Hamiltonian system for linear hyperbolic systems, which include the rotating shallow water equations, the acoustic and Maxwell equations. These equations have a Hamiltonian structure with a bilinear Poisson bracket, and as a consequence the phase-space structure, "mass" and energy are preserved. We discretize the bilinear Poisson bracket in each element with discontinuous elements and introduce numerical fluxes via integration by parts while preserving the skew-symmetry of the bracket. This automatically results in a mass and energy conservative discretization. When combined with a symplectic time integration method, energy is approximately conserved and shows no drift. For comparison, the discontinuous Galerkin method for this problem is also used. A variety numerical examples is shown to illustrate the accuracy and capability of the new method.
\end{abstract}

Keywords Rotating shallow water equations - Acoustic equations · Maxwell equations · Hamiltonian dynamics · Discontinuous Galerkin method · Numerical flux

\section{Introduction}

Many space-time dynamical systems in physics and mathematics are Hamiltonian and have conservation laws associated with their Hamiltonian formulation. Preservation of the Hamiltonian formulation in the discretizations of these systems is especially desirable in long-time

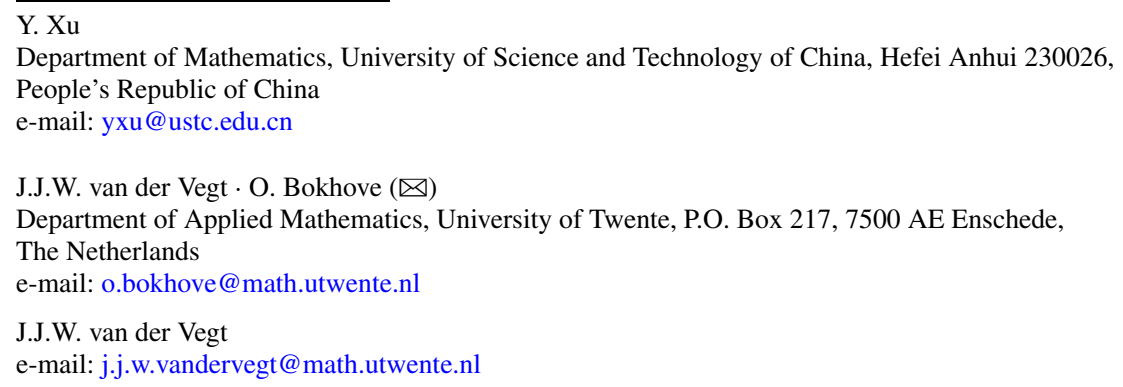


predictions where conservation laws constrain the dynamics whereas dissipative discretizations do not. A space-time Hamiltonian system consists of the dynamics of an arbitrary functional of the variables, a (generalized) Poisson bracket and a Hamiltonian, see e.g. [12]. This (generalized) Poisson bracket is skew-symmetric and satisfies the Jacobi identity [10, 11]. Typically the formulation deals with functionals. Here, we restrict attention to a generalized Hamiltonian formulation for linear hyperbolic systems including the rotating linearized shallow water equations, the acoustic and Maxwell's equations. These linear hyperbolic equations generally involve given functions of space, representing the spatial variation of material properties of the associated physical system.

The Hamiltonian formulation of our generalized system guarantees that energy, "mass" and phase-space structure are preserved (see [5]). The standard discontinuous Galerkin finite element method, however, fails to conserve energy when the material properties are spatially varying, while discrete energy conservation has been obtained for constant coefficients [14]. This motivated us to derive a weak formulation and corresponding discontinuous finite element discretization directly based on discretizing the generalized Poisson bracket in the Hamiltonian formulation. Since this results in a skew-symmetric spatial discretization, energy conservation is directly ensured. Furthermore, the equivalent "mass" field in the problem is conserved. By additional use of symplectic splitting methods for the time discretization, the phase space structure is preserved while the energy oscillates weakly around its initial value [5], without drift.

To investigate the strength of our Hamiltonian discontinuous finite element formulation we contrast it with the classical discontinuous Galerkin (DG) formulation involving an alternating numerical flux [14]. This classical DG method is a class of finite element methods using completely discontinuous piecewise polynomial spaces for the numerical solution and the test functions in the spatial variables, usually coupled with an explicit and nonlinearly stable high order Runge-Kutta time discretization [13], first developed in [3, 4].

The standard DG finite element method with an alternating numerical flux and our new DG finite element method based on the skew-symmetric Hamiltonian formulation coincide when the material functions are constant. It demonstrates that the alternating flux can be interpreted as a skew-symmetric Hamiltonian flux. In contrast, the skew-symmetric flux becomes essential to conserve energy and phase space volume in the important case of spatially varying material functions.

The outline of our article is as follows. In Sect. 2, we present the generalized linear hyperbolic system and its Hamiltonian formulation. In Sect. 3, we derive the discontinuous finite element discretization for the generalized linear hyperbolic equations and the ensuing discrete skew-symmetric bracket. For comparison, we also give the DG method for the generalized linear hyperbolic equations. The symplectic splitting method and classical Runge-Kutta time discretizations used are presented in Sect. 4. Section 5 contains numerical results for the linear problems to demonstrate the accuracy and capabilities of the new method. Concluding remarks are given in Sect. 6.

\section{Hamiltonian Formulation}

\subsection{General Formulation}

We consider the linear hyperbolic system of equations

$$
\frac{\partial \boldsymbol{v}}{\partial t}+\mathcal{D}(C \eta)+f \boldsymbol{v}^{\perp}=0
$$




$$
\frac{\partial \eta}{\partial t}+\mathcal{D} \cdot(B \boldsymbol{v})=0, \quad \forall(x, y) \in \Omega \subseteq \mathbb{R}^{2},
$$

with the two-dimensional vector field $\boldsymbol{v}=\boldsymbol{v}(x, y, t)=(u, v)^{T}, \boldsymbol{v}^{\perp}=(-v, u)^{T}$ and the scalar function $\eta=\eta(x, y, t)$, depending on spatial coordinates $x, y$ and time $t$; and, the given functions $B=B(x, y)>0, C=C(x, y)>0$ and $f=f(x, y)$. The operator $\mathcal{D}$ is either the differential operator $\nabla=\left(\frac{\partial}{\partial x}, \frac{\partial}{\partial y}\right)^{T}$, or $-\nabla^{\perp}=\left(\frac{\partial}{\partial y},-\frac{\partial}{\partial x}\right)^{T}$.

The domain $\Omega$ has a boundary $\partial \Omega$, which is subdivided into boundary segments, at which boundary conditions are specified, such as periodic boundary conditions and/or solid walls. At solid walls $\partial \Omega_{s} \subseteq \partial \Omega$ the boundary condition

$$
\mathcal{N} \cdot v=0
$$

is imposed. Here the vector $\mathcal{N}$ is either the normal vector $\boldsymbol{n}=\left(n_{x}, n_{y}\right)^{T}$ or the tangential vector $\boldsymbol{n}^{\perp}=\left(n_{y},-n_{x}\right)^{T}$ at the boundary of $\partial \Omega_{s}$, depending if the differential operator $\mathcal{D}$ is equal to $\mathcal{D}=\nabla$ or $\mathcal{D}=-\nabla^{\perp}$, respectively. The system (2.1) is completed with the initial conditions $\boldsymbol{v}(x, y, 0)=\boldsymbol{v}_{0}(x, y)$ and $\eta(x, y, 0)=\eta_{0}(x, y)$. Additional consistency requirements emerge because (2.2) must be preserved in time.

The linear system of (2.1) has a Hamiltonian formulation, see e.g. [1, 12], which can be expressed using the Poisson bracket $\{\cdot, \cdot\}_{1}$ as

$$
\frac{\mathrm{d} \mathcal{F}}{\mathrm{d} t}=\{\mathcal{F}, \mathcal{H}\}_{1}=\int_{\Omega}\left(\frac{f}{B} \frac{\delta \mathcal{F}}{\delta \boldsymbol{v}}^{\perp} \cdot \frac{\delta \mathcal{H}}{\delta \boldsymbol{v}}+\left(\mathcal{D} \cdot \frac{\delta \mathcal{F}}{\delta \boldsymbol{v}}\right) \frac{\delta \mathcal{H}}{\delta \eta}-\frac{\delta \mathcal{F}}{\delta \eta}\left(\mathcal{D} \cdot \frac{\delta \mathcal{H}}{\delta \boldsymbol{v}}\right)\right) \mathrm{d} \Omega
$$

with Hamiltonian

$$
\mathcal{H}=\int_{\Omega}\left(\frac{1}{2} B|\boldsymbol{v}|^{2}+\frac{1}{2} C \eta^{2}\right) \mathrm{d} \Omega .
$$

The functional derivatives of the Hamiltonian are defined as

$$
\delta \mathcal{H}:=\lim _{\epsilon \rightarrow 0} \frac{\mathcal{H}[\boldsymbol{v}+\epsilon \delta \boldsymbol{v}, \eta+\epsilon \delta \eta]-\mathcal{H}[\boldsymbol{v}, \eta]}{\epsilon}:=\int_{\Omega}\left(\frac{\delta \mathcal{H}}{\delta \boldsymbol{v}} \cdot \delta \boldsymbol{v}+\frac{\delta \mathcal{H}}{\delta \eta} \delta \eta\right) \mathrm{d} \Omega .
$$

Hence, it follows from (2.4) and (2.5) that

$$
\delta \mathcal{H}=\int_{\Omega}(B \boldsymbol{v} \cdot \delta \boldsymbol{v}+C \eta \delta \eta) \mathrm{d} \Omega
$$

and by using (2.5) with (2.6) we obtain the functional derivatives

$$
\frac{\delta \mathcal{H}}{\delta \boldsymbol{v}}=B \boldsymbol{v} \quad \text { and } \quad \frac{\delta \mathcal{H}}{\delta \eta}=C \eta .
$$

The equations for the velocity field $\boldsymbol{v}$, given by (2.1a), are obtained if we choose the functional $\mathcal{F}$ in $(2.3)$ as:

$$
\mathcal{F}[\boldsymbol{v}]=\int_{\Omega} \boldsymbol{w}_{\boldsymbol{v}}(\boldsymbol{x}) \cdot \boldsymbol{v}(\boldsymbol{x}, t) \mathrm{d} \Omega,
$$

with $\boldsymbol{w}_{v}$ arbitrary functions which satisfy the condition $\mathcal{N} \cdot \boldsymbol{w}_{v}=0$ at $\partial \Omega_{s}$. Similarly, the equation for $\eta$, given by (2.1b), is obtained if we choose the functional $\mathcal{F}$ with $w_{\eta}$ arbitrary functions as

$$
\mathcal{F}[\eta]=\int_{\Omega} w_{\eta}(\boldsymbol{x}) \eta(\boldsymbol{x}, t) \mathrm{d} \Omega
$$


The bracket $\{\mathcal{F}, \mathcal{H}\}_{1}$ is seen to be skew-symmetric and also satisfies the Jacobi identity

$$
\left\{\mathcal{K},\{\mathcal{F}, \mathcal{G}\}_{1}\right\}_{1}+\left\{\mathcal{F},\{\mathcal{G}, \mathcal{K}\}_{1}\right\}_{1}+\left\{\mathcal{G},\{\mathcal{K}, \mathcal{F}\}_{1}\right\}_{1}=0
$$

for arbitrary functionals $\mathcal{F}, \mathcal{G}$ and $\mathcal{K}$. The skew-symmetry of the bracket in (2.3) guarantees energy conservation, since

$$
\frac{\mathrm{d} \mathcal{H}}{\mathrm{d} t}=\{\mathcal{H}, \mathcal{H}\}_{1}=0,
$$

and mass conservation follows after inserting the variational derivatives into the Poisson bracket and using the boundary conditions

$$
\frac{\mathrm{d} \mathcal{M}}{\mathrm{d} t}=\{\mathcal{M}, \mathcal{H}\}_{1}=0 \quad \text { with } \mathcal{M}=\int_{\Omega} \eta \mathrm{d} \Omega .
$$

An alternative form of bracket $\{\cdot, \cdot\}_{1}$ appears after integration by parts and using the boundary conditions, i.e.

$$
\frac{\mathrm{d} \mathcal{F}}{\mathrm{d} t}=\{\mathcal{F}, \mathcal{H}\}_{2}:=\int_{\Omega}\left(\frac{f}{B} \frac{\delta \mathcal{F}^{\perp}}{\delta \boldsymbol{v}} \cdot \frac{\delta \mathcal{H}}{\delta \boldsymbol{v}}-\frac{\delta \mathcal{F}}{\delta \boldsymbol{v}} \cdot\left(\mathcal{D} \frac{\delta \mathcal{H}}{\delta \eta}\right)-\frac{\delta \mathcal{F}}{\delta \eta}\left(\mathcal{D} \cdot \frac{\delta \mathcal{H}}{\delta \boldsymbol{v}}\right)\right) \mathrm{d} \Omega .
$$

The natural boundary conditions for $\boldsymbol{v}$ at solid walls extend to the functional derivatives; they are for arbitrary $\mathcal{F}$

$$
\mathcal{N} \cdot \frac{\delta \mathcal{F}}{\delta \boldsymbol{v}}=0
$$

The skew-symmetric nature is now hidden in (2.9) in contrast to the form of the bracket (2.3).

Although $\{\cdot, \cdot\}_{1}$ directly results in a skew-symmetric discrete bracket it does not directly show a relation to the classical DG method, which is based on a weak formulation of the partial differential equations, cf. [4]. This is more clear if we use the discrete form of $\{\cdot, \cdot\}_{2}$, see Sect. 3. In particular, we will show that for certain numerical fluxes the spatial discretization of both brackets coincides, and then both approaches guarantee discrete energy conservation.

\subsection{Applications}

In this section, we will discuss several important examples of Hamiltonian systems for linear hyperbolic systems which can be discretized with the Hamiltonian discontinuous finite element discretization derived later.

\subsubsection{Rotating Shallow Water Equations}

The linear rotating shallow water equations are a special case of system (2.1) with $\mathcal{D}=\nabla$, $B(x, y)=D(x, y)$ the given rest depth, $C(x, y)=g$ the constant gravitational acceleration, and $f=f(x, y)$ the given Coriolis parameter. Hence, the resulting rotating shallow water equations are

$$
\frac{\partial \boldsymbol{v}}{\partial t}+\nabla(g \eta)+f \boldsymbol{v}^{\perp}=0, \quad \frac{\partial \eta}{\partial t}+\nabla \cdot(D \boldsymbol{v})=0,
$$


where $\boldsymbol{v}$ is the velocity and $\eta$ is the water depth. Slip flow implies no flow through solid walls: $\boldsymbol{n} \cdot \boldsymbol{v}=0$, and when $f \neq 0$ geostrophic balance holds at these solid boundaries, $\boldsymbol{n} \cdot \nabla(g \eta)+f \boldsymbol{n} \cdot \boldsymbol{v}^{\perp}=0$, such that the flow tangential to the wall is balanced by the normal gradient of the geopotential $g \eta$. When $f=0$ the usual Neumann relation $\boldsymbol{n} \cdot \nabla \eta=0$ at a solid-wall boundary results.

The linear rotating shallow water equations have the following Hamiltonian formulation

$$
\frac{\mathrm{d} \mathcal{F}}{\mathrm{d} t}=\left\{\mathcal{F}, \mathcal{H}_{l}\right\}_{1}=\int_{\Omega}\left(\frac{f}{D} \frac{\delta \mathcal{F}^{\perp}}{\delta \boldsymbol{v}} \cdot \frac{\delta \mathcal{H}_{l}}{\delta \boldsymbol{v}}+\left(\nabla \cdot \frac{\delta \mathcal{F}}{\delta \boldsymbol{v}}\right) \frac{\delta \mathcal{H}_{l}}{\delta \eta}-\frac{\delta \mathcal{F}}{\delta \eta}\left(\nabla \cdot \frac{\delta \mathcal{H}_{l}}{\delta \boldsymbol{v}}\right)\right) \mathrm{d} \Omega,
$$

with Hamiltonian

$$
\mathcal{H}_{l}=\int_{\Omega}\left(\frac{1}{2} D|\boldsymbol{v}|^{2}+\frac{1}{2} g \eta^{2}\right) \mathrm{d} \Omega .
$$

Its functional derivatives are

$$
\frac{\delta \mathcal{H}_{l}}{\delta \boldsymbol{v}}=D \boldsymbol{v} \quad \text { and } \quad \frac{\delta \mathcal{H}_{l}}{\delta \eta}=g \eta .
$$

\subsubsection{D Maxwell Equations}

Another application of (2.1) concerns the two-dimensional Maxwell equations with $\boldsymbol{v}=$ $\boldsymbol{H}=\left(H_{x}, H_{y}\right)^{T}$ the magnetic field, $\eta=E_{z}$ the electric field, and $\mathcal{D}=-\nabla^{\perp}, C=\mu^{-1}$, $B=\epsilon^{-1}$ and $f=0$. The two-dimensional Maxwell equations are defined as

$$
\frac{\partial \boldsymbol{H}}{\partial t}=\nabla^{\perp}\left(\mu^{-1} E_{z}\right), \quad \frac{\partial E_{z}}{\partial t}=\nabla^{\perp} \cdot\left(\epsilon^{-1} \boldsymbol{H}\right),
$$

where $\mu$ is the magnetic permeability and $\epsilon$ is the dielectric permittivity. At solid walls $\boldsymbol{n} \cdot \boldsymbol{H}^{\perp}=0$.

The Maxwell equations have the following Hamiltonian formulation

$$
\frac{\mathrm{d} \mathcal{F}}{\mathrm{d} t}=\left\{\mathcal{F}, \mathcal{H}_{m}\right\}_{1}=\int_{\Omega}\left(-\left(\nabla^{\perp} \cdot \frac{\delta \mathcal{F}}{\delta \boldsymbol{H}}\right) \frac{\delta \mathcal{H}_{m}}{\delta E_{z}}+\frac{\delta \mathcal{F}}{\delta E_{z}}\left(\nabla^{\perp} \cdot \frac{\delta \mathcal{H}_{m}}{\delta \boldsymbol{H}}\right)\right) \mathrm{d} \Omega,
$$

with Hamiltonian

$$
\mathcal{H}_{m}=\int_{\Omega}\left(\frac{1}{2} \epsilon^{-1}|\boldsymbol{H}|^{2}+\frac{1}{2} \mu^{-1} E_{z}^{2}\right) \mathrm{d} \Omega
$$

Its functional derivatives are

$$
\frac{\delta \mathcal{H}_{m}}{\delta \boldsymbol{H}}=\epsilon^{-1} \boldsymbol{H} \quad \text { and } \quad \frac{\delta \mathcal{H}_{m}}{\delta E_{z}}=\mu^{-1} E_{z} .
$$

\subsubsection{Acoustic Equations}

The two-dimensional acoustic equations [9] arise from (2.1) when $v=(u, v)^{T}$ is taken as the velocity field, $\eta=\rho$ as density, and $\mathcal{D}=\nabla, C=c_{0}^{2} / \rho_{0}$ with $B=\rho_{0}$ as reference density, and $f=0$. The two-dimensional acoustic equations then become

$$
\frac{\partial \boldsymbol{v}}{\partial t}+\nabla\left(\frac{c_{0}^{2}}{\rho_{0}} \rho\right)=0, \quad \frac{\partial \rho}{\partial t}+\nabla \cdot\left(\rho_{0} \boldsymbol{v}\right)=0 .
$$


Slip flow again implies no flow through solid walls: $\boldsymbol{n} \cdot \boldsymbol{v}=0$. The acoustic equations have the following Hamiltonian formulation

$$
\frac{\mathrm{d} \mathcal{F}}{\mathrm{d} t}=\left\{\mathcal{F}, \mathcal{H}_{a}\right\}_{1}=\int_{\Omega}\left(\left(\nabla \cdot \frac{\delta \mathcal{F}}{\delta \boldsymbol{v}}\right) \frac{\delta \mathcal{H}_{a}}{\delta \rho}-\frac{\delta \mathcal{F}}{\delta \rho}\left(\nabla \cdot \frac{\delta \mathcal{H}_{a}}{\delta \boldsymbol{v}}\right)\right) \mathrm{d} \Omega,
$$

with Hamiltonian

$$
\mathcal{H}_{a}=\int_{\Omega}\left(\frac{1}{2} \rho_{0}|\boldsymbol{v}|^{2}+\frac{1}{2} \frac{c_{0}^{2}}{\rho_{0}} \rho^{2}\right) \mathrm{d} \Omega,
$$

and functional derivatives

$$
\frac{\delta \mathcal{H}_{a}}{\delta \boldsymbol{v}}=\rho_{0} \boldsymbol{v} \quad \text { and } \quad \frac{\delta \mathcal{H}_{a}}{\delta \rho}=\frac{c_{0}^{2}}{\rho_{0}} \rho .
$$

\section{Discrete Hamiltonian Formulation}

In this section, we will derive a spatial Hamiltonian discontinuous finite element discretization for the Hamiltonian system (2.3) and (2.4). It thus guarantees conservation of energy and phase space volume by default. It will be shown that it coincides with a particular discretization of (2.9) with Hamiltonian (2.4). For comparison, we will also give the DG discretization for the generalized linear hyperbolic equations (2.1).

\subsection{Notation}

Let $\mathcal{T}_{h}$ denote a tessellation of $\Omega$ with shape-regular elements $K$. Let $\Gamma$ denote the set of all edges in the tessellation $\mathcal{T}_{h}$, with $\Gamma_{i}$ the set of interior edges and $\Gamma_{b}$ the set of edges at the domain boundary.

In order to describe the flux functions we need to introduce some notation. Let $e$ be an edge shared by the "left" and "right" elements $K_{L}$ and $K_{R}$. Define the normal vectors $n_{L}$ and $n_{R}$ on $e$ pointing exterior to $K_{L}$ and $K_{R}$, respectively. When $e$ lies on the domain boundary, we adopt the convention that $K_{L}$ lies inside $\Omega$. If $\psi$ is a function on $K_{L}$ and $K_{R}$, but possibly discontinuous across $e$, let $\psi_{L}=\left.\left(\left.\psi\right|_{K_{L}}\right)\right|_{e}$ and $\psi_{R}=\left.\left(\left.\psi\right|_{K_{R}}\right)\right|_{e}$ denote the left and right trace, respectively.

Let $\mathcal{P}^{p}(K)$ be the space of polynomials of degree at most $p$ on $K \in \mathcal{T}_{h}$, with $p \geq 0$. The finite element spaces $V_{h}$ and $W_{h}$ are denoted by

$$
\begin{aligned}
V_{h} & =\left\{\psi \in L^{2}(\Omega):\left.\psi\right|_{K} \in \mathcal{P}^{p}(K), \forall K \in \mathcal{T}_{h}\right\}, \\
W_{h} & =\left\{\psi \in\left(L^{2}(\Omega)\right)^{2}:\left.\psi\right|_{K} \in\left(\mathcal{P}^{p}(K)\right)^{2}, \forall K \in \mathcal{T}_{h},\left.\mathcal{N} \cdot \boldsymbol{\psi}\right|_{\partial \Omega_{s}}=0\right\} .
\end{aligned}
$$

The number of degrees of freedom on an element is denoted by $N_{K}=\operatorname{dim}\left(\mathcal{P}^{p}(K)\right)$.

3.2 Discrete Hamiltonian Formulation and Variational Derivatives

Consider the linear system (2.1) rewritten in the form

$$
\frac{\partial \boldsymbol{v}}{\partial t}+\mathcal{D} r+\frac{f}{B} \mathbf{Q}^{\perp}=0 \quad \text { and } \quad \frac{\partial \eta}{\partial t}+\mathcal{D} \cdot \mathbf{Q}=0
$$

with $\mathbf{Q}=B \boldsymbol{v}$ and $r=C \eta$. 
Energy conservation follows by multiplying the first equation in (3.1) by $\mathbf{Q}$ and the second equation in (3.1) by $r$, integration over the domain $\Omega$, applying Gauss' law and using the boundary conditions, i.e.

$$
\frac{\mathrm{d}}{\mathrm{d} t} \frac{1}{2} \int_{\Omega} B|\boldsymbol{v}|^{2}+C \eta^{2} \mathrm{~d} \Omega=-\int_{\Omega} \mathcal{D} \cdot(\mathbf{Q} r) \mathrm{d} \Omega=0 .
$$

The crucial point in a corresponding discontinuous Hamiltonian discretization is to consider $\mathbf{Q}$ and $r$ as additional variables, linked to $B \boldsymbol{v}$ and $C \eta$ via a projection onto the finite element space.

In the discrete Hamiltonian formulation we will use $H$ to denote the discrete approximation of $\mathcal{H}$. The discrete Hamiltonian is

$$
H=\frac{1}{2} \sum_{K} \int_{K}\left(B_{h}\left|\boldsymbol{v}_{h}\right|^{2}+C_{h} \eta_{h}^{2}\right) \mathrm{d} \Omega,
$$

where $\eta_{h} \in V_{h}$ and $\boldsymbol{v}_{h} \in V_{h} \times V_{h}$. In contrast to the continuous case, the variational derivatives are not equal in the strong sense, but only in a weak sense

$$
\frac{\delta H}{\delta \boldsymbol{v}_{h}}=\mathbf{Q}_{h} \neq B_{h} \boldsymbol{v}_{h}, \quad \frac{\delta H}{\delta \eta_{h}}=r_{h} \neq C_{h} \eta_{h},
$$

where $r_{h} \in V_{h}$ and $\mathbf{Q}_{h} \in V_{h} \times V_{h}$.

The Hamiltonian discretization automatically does this because $\mathbf{Q}_{h}=\delta H / \delta \boldsymbol{v}_{h}$ and $r_{h}=$ $\delta H / \delta \eta_{h}$. Consequently, we should use $\mathbf{Q}_{h}$ and $r_{h}$ in the discretization and not $B_{h} \boldsymbol{v}_{h}$ and $C_{h} \eta_{h}$ as the former lie in $V_{h} \times V_{h}$ and $V_{h}$, respectively, while the latter do not. We will show that the functional derivatives in the Hamiltonian formulation project onto the Galerkin space, and also that the Hamiltonian remains positive.

For the discretization of the velocity (2.1a) we consider the functional $F\left[\boldsymbol{v}_{h}\right]=$ $\int_{\Omega} \boldsymbol{v}_{h} \cdot \boldsymbol{\psi} \mathrm{d} \Omega$, with $\psi \in W_{h}$ arbitrary test functions. Using the definition of the functional derivatives

$$
\delta F:=\lim _{\epsilon \rightarrow 0} \frac{F\left[\boldsymbol{v}_{h}+\epsilon \delta \boldsymbol{v}_{h}\right]-F\left[\boldsymbol{v}_{h}\right]}{\epsilon}=\int_{\Omega} \boldsymbol{\psi} \cdot \delta \boldsymbol{v}_{h} \mathrm{~d} \Omega,
$$

we obtain

$$
\frac{\delta F}{\delta \boldsymbol{v}_{h}}=\psi
$$

The test function $\psi$ is taken from the space $W_{h}$ and not from $V_{h} \times V_{h}$, since the functional derivative of $F\left[\boldsymbol{v}_{h}\right]$ must satisfy the condition (2.10) at the boundary $\Omega_{s}$. This implies that

$$
\mathcal{N} \cdot \frac{\delta F}{\delta \boldsymbol{v}_{h}}=\mathcal{N} \cdot \boldsymbol{\psi}=0
$$

Hence the test functions $\psi$ at the domain boundary must have either a zero normal or tangential component depending on the choice of the operator $\mathcal{D}$, viz. $\mathcal{D}=\nabla$ or $\mathcal{D}=-\nabla^{\perp}$.

Likewise, for the discretization of the equation for $\eta$, given by $(2.1 \mathrm{~b})$, we set the functional $F$ equal to $F\left[\eta_{h}\right]=\int_{\Omega} \eta_{h} \phi \mathrm{d} \Omega$, with $\phi \in V_{h}$ arbitrary test functions, and we obtain the functional derivative

$$
\frac{\delta F}{\delta \eta_{h}}=\phi
$$




\subsection{The Discontinuous Hamiltonian Formulation}

In this section we will derive a discrete formulation for the Hamiltonian system (2.3)-(2.4). We will start with bracket $\{\cdot, \cdot\}_{2}$, defined in (2.9), and by choosing proper numerical fluxes we can demonstrate the skew-symmetry of the discrete bracket when using discontinuous basis functions. This then automatically implies conservation of mass and energy at the discrete level. The discrete form of formulation (2.9) is obtained by introducing the tessellation $\mathcal{T}_{h}$ of $\Omega$ and the discrete approximations of the functionals $\mathcal{F}$ and $\mathcal{H}$. After integration by parts over each element $K \in \mathcal{T}_{h}$, we obtain

$$
\begin{aligned}
\frac{\mathrm{d} F}{\mathrm{~d} t}= & \{F, H\}_{2} \\
= & \sum_{K \in \mathcal{T}_{h}} \int_{K}\left(\frac{f_{h}}{B_{h}} \frac{\delta F^{\perp}}{\delta \boldsymbol{v}_{h}} \cdot \frac{\delta H}{\delta \boldsymbol{v}_{h}}\right) \mathrm{d} \Omega+\sum_{K \in \mathcal{T}_{h}} \int_{K}\left(\left(\mathcal{D} \cdot \frac{\delta F}{\delta \boldsymbol{v}_{h}}\right) \frac{\delta H}{\delta \eta_{h}}+\left(\mathcal{D} \frac{\delta F}{\delta \eta_{h}}\right) \cdot \frac{\delta H}{\delta \boldsymbol{v}_{h}}\right) \mathrm{d} \Omega \\
& -\sum_{K \in \mathcal{T}_{h}} \int_{\partial K}\left(\mathcal{N} \cdot \frac{\delta F}{\delta \boldsymbol{v}_{h}} \frac{\widehat{\delta H}}{\delta \eta_{h}}+\widehat{\mathcal{N} \cdot \frac{\delta H}{\delta \boldsymbol{v}_{h}}} \frac{\delta F}{\delta \eta_{h}}\right) \mathrm{d} S,
\end{aligned}
$$

where the numerical fluxes $\delta \widehat{H / \delta \eta}_{h}$ and $\mathcal{N} \widehat{\cdot \delta H /} \delta v_{h}$ are introduced to account for the multivalued traces of $\delta H / \delta \eta_{h}$ and $\mathcal{N} \cdot \delta H / \delta \boldsymbol{v}_{h}$ at the element boundaries $\partial K$. Since all derivative terms in the Poisson bracket $\{\cdot, \cdot\}_{2}$ are on the Hamilton functional, the numerical flux at the element boundaries can be chosen using the alternating numerical flux proposed in [14]. This procedure is different for the Poisson bracket $\{\cdot, \cdot\}_{1},(2.3)$, because the functional derivatives of $F,(3.6)$ and (3.8), are arbitrary test functions.

By choosing the functional $F$ alternatively as

$$
\int_{\Omega} \boldsymbol{v}_{h} \cdot \boldsymbol{\psi} \mathrm{d} \Omega \text { and } \int_{\Omega} \eta_{h} \phi \mathrm{d} \Omega,
$$

introducing these relations into (3.9) and using the discrete variational derivatives (3.4), (3.6) and (3.8), the following discrete formulation for (2.1) emerges:

Find a $\boldsymbol{v}_{h} \in V_{h} \times V_{h}$ and $\eta_{h} \in V_{h}$, such that for all $\psi \in W_{h}$ and $\phi \in V_{h}$ the following relation is satisfied:

$$
\begin{aligned}
\int_{K} \frac{\partial \boldsymbol{v}_{h}}{\partial t} \cdot \boldsymbol{\psi} \mathrm{d} \Omega & =\int_{K}\left(-\frac{f_{h}}{B_{h}} \mathbf{Q}_{h}^{\perp} \cdot \boldsymbol{\psi}+r_{h} \mathcal{D} \cdot \boldsymbol{\psi}\right) \mathrm{d} \Omega-\int_{\partial K} \widehat{r_{h}} \mathcal{N} \cdot \boldsymbol{\psi} \mathrm{d} S, \\
\int_{K} \frac{\partial \eta_{h}}{\partial t} \phi \mathrm{d} \Omega & =\int_{K} \mathbf{Q}_{h} \cdot \mathcal{D} \phi \mathrm{d} \Omega-\int_{\partial K} \widehat{\mathcal{N} \cdot \mathbf{Q}_{h}} \phi \mathrm{d} S
\end{aligned}
$$

where $\mathbf{Q}_{h} \in V_{h} \times V_{h}$ and $r_{h} \in V_{h}$ are obtained from the relations

$$
\begin{aligned}
& \int_{K} \mathbf{Q}_{h} \cdot \boldsymbol{\phi} \mathrm{d} \Omega=\int_{K} B_{h} \boldsymbol{v}_{h} \cdot \boldsymbol{\phi} \mathrm{d} \Omega, \quad \forall \boldsymbol{\phi} \in V_{h} \times V_{h}, \\
& \int_{K} r_{h} \phi \mathrm{d} x \mathrm{~d} y=\int_{K} C_{h} \eta_{h} \phi \mathrm{d} \Omega, \quad \forall \phi \in V_{h} .
\end{aligned}
$$


We choose the following alternating numerical fluxes at edges $e \in \Gamma_{i}$

$$
\begin{aligned}
\frac{\widehat{\delta H}}{\delta \eta_{h}} & =\widehat{r}_{h}=\theta \frac{\delta H}{\delta \eta_{h}^{L}}+(1-\theta) \frac{\delta H}{\delta \eta_{h}^{R}}, \\
\widehat{\mathcal{N} \cdot \frac{\delta H}{\delta \boldsymbol{v}_{h}}} & ={\widehat{\mathcal{N}} \cdot \mathbf{Q}_{h}}=(1-\theta) \mathcal{N} \cdot \frac{\delta H}{\delta \boldsymbol{v}_{h}^{L}}+\theta \mathcal{N} \cdot \frac{\delta H}{\delta \boldsymbol{v}_{h}^{R}}, \quad 0 \leq \theta \leq 1,
\end{aligned}
$$

where we have uniquely defined a left and right side with a positive orientation of the edge numbering per element. Here and hereafter $\mathcal{N}=\mathcal{N}^{L}$. At edges $e \in \Gamma_{b}$ at the domain boundary $\partial \Omega_{s}$, we introduce the boundary conditions (2.2) and (2.10)

$$
\widehat{\mathcal{N} \cdot \frac{\delta H}{\delta \boldsymbol{v}_{h}}}=\mathcal{N} \cdot \mathbf{Q}_{h}=0 \quad \text { and } \quad \widehat{\mathcal{N} \cdot \frac{\delta F}{\delta \boldsymbol{v}_{h}}}=\mathcal{N} \cdot \boldsymbol{\psi}=0 .
$$

After the introduction of the numerical fluxes (3.14) and using the fact that each edge occurs twice in the summation over all elements we can rewrite the discrete form of (3.9) as

$$
\begin{aligned}
\frac{\mathrm{d} F}{\mathrm{~d} t}=\{ & F, H\}_{2}=\sum_{K \in \mathcal{T}_{h}} \int_{K}\left(\frac{f_{h}}{B_{h}} \frac{\delta F^{\perp}}{\delta \boldsymbol{v}_{h}} \cdot \frac{\delta H}{\delta \boldsymbol{v}_{h}}\right) \mathrm{d} \Omega \\
& +\sum_{K \in \mathcal{T}_{h}} \int_{K}\left(\left(\mathcal{D} \cdot \frac{\delta F}{\delta \boldsymbol{v}_{h}}\right) \frac{\delta H}{\delta \eta_{h}}+\left(\mathcal{D} \frac{\delta F}{\delta \eta_{h}}\right) \cdot \frac{\delta H}{\delta \boldsymbol{v}_{h}}\right) \mathrm{d} \Omega \\
& +\sum_{e \in \Gamma_{i}} \int_{e} \mathcal{N} \cdot\left(\frac{\delta F}{\delta \boldsymbol{v}_{h}^{R}}-\frac{\delta F}{\delta \boldsymbol{v}_{h}^{L}}\right)\left(\theta \frac{\delta H}{\delta \eta_{h}^{L}}+(1-\theta) \frac{\delta H}{\delta \eta_{h}^{R}}\right) \\
& +\left(\frac{\delta F}{\delta \eta_{h}^{R}}-\frac{\delta F}{\delta \eta_{h}^{L}}\right) \mathcal{N} \cdot\left(\theta \frac{\delta H}{\delta \boldsymbol{v}_{h}^{R}}+(1-\theta) \frac{\delta H}{\delta \boldsymbol{v}_{h}^{L}}\right) \mathrm{d} S .
\end{aligned}
$$

\subsection{The Skew-Symmetry of the Discrete Bracket}

The discrete bracket (3.15) apparently lacks the skew-symmetry, which seems to withhold an immediate proof of energy conservation. The skew-symmetry of the discrete bracket can, however, be demonstrated using a discretization of the skew-symmetric bracket given by (2.3), and related to the discrete bracket (3.15). This approach will also indicate how to obtain a suitable discretization for bracket $\{\cdot, \cdot\}_{1}$. The equivalence of these two Hamiltonian discretizations giving (3.10)-(3.11) automatically leads to energy conservation at the discrete level.

The discretization of the bracket $\{\cdot, \cdot\}_{1}$ in $(2.3)$ yields

$$
\begin{aligned}
\{F, H\}_{1}= & \sum_{K \in \mathcal{T}_{h}} \int_{K}\left(\frac{f_{h}}{B_{h}} \frac{\delta F^{\perp}}{\delta \boldsymbol{v}_{h}} \cdot \frac{\delta H}{\delta \boldsymbol{v}_{h}}\right) \mathrm{d} \Omega \\
& +\sum_{K \in \mathcal{T}_{h}} \int_{K}\left(-\frac{\delta F}{\delta \boldsymbol{v}_{h}} \cdot\left(\mathcal{D} \frac{\delta H}{\delta \eta_{h}}\right)+\left(\mathcal{D} \frac{\delta F}{\delta \eta_{h}}\right) \cdot \frac{\delta H}{\delta \boldsymbol{v}_{h}}\right) \mathrm{d} \Omega \\
& +\sum_{K \in \mathcal{T}_{h}} \int_{\partial K}\left(\widehat{\mathcal{N} \cdot \frac{\delta F}{\delta \boldsymbol{v}_{h}}} \frac{\delta H}{\delta \eta_{h}}-\widehat{\mathcal{N} \cdot \frac{\delta H}{\delta \boldsymbol{v}_{h}}} \frac{\delta F}{\delta \eta_{h}}\right) \mathrm{d} S
\end{aligned}
$$


where the numerical fluxes $\mathcal{\mathcal { N }} \widehat{\cdot \delta F / \delta} \boldsymbol{v}$ and $\mathcal{N} \widehat{\cdot \delta H / \delta} \boldsymbol{v}_{h}$ are introduced. When the numerical flux $\mathcal{N} \widehat{\cdot \delta F / \delta} \boldsymbol{v}_{h}$ is chosen the same as for $\mathcal{N} \cdot \widehat{\delta H /} \delta \boldsymbol{v}_{h}$, the discrete bracket is skewsymmetric. Energy and mass are then automatically conserved at the discrete level.

For the specific choice of the numerical flux given by (3.14), we obtain for bracket $\{\cdot, \cdot\}_{1}$ at interior edges $e \in \Gamma_{i}$

$$
\begin{aligned}
& \widehat{\mathcal{N} \cdot \frac{\delta F}{\delta \boldsymbol{v}_{h}}}=(1-\theta) \mathcal{N} \cdot \frac{\delta F}{\delta \boldsymbol{v}_{h}^{L}}+\theta \mathcal{N} \cdot \frac{\delta F}{\delta \boldsymbol{v}_{h}^{R}}, \\
& \widehat{\mathcal{N} \cdot \frac{\delta H}{\delta \boldsymbol{v}_{h}}}=(1-\theta) \mathcal{N} \cdot \frac{\delta H}{\delta \boldsymbol{v}_{h}^{L}}+\theta \mathcal{N} \cdot \frac{\delta H}{\delta \boldsymbol{v}_{h}^{R}}, \quad 0 \leq \theta \leq 1 .
\end{aligned}
$$

At the domain boundary $\partial \Omega_{s}$, we must satisfy for edges $e \in \Gamma_{b}$, the condition (2.2)

$$
\widehat{\mathcal{N} \cdot \frac{\delta H}{\delta \boldsymbol{v}_{h}}}=\mathcal{N} \cdot \mathbf{Q}_{h}=0 .
$$

In order to ensure the skew-symmetry of the bracket, this implies the following boundary condition at $\partial \Omega_{s}$ on the functional derivative of $\mathcal{F}$

$$
\widehat{\mathcal{N} \cdot \frac{\delta F}{\delta \boldsymbol{v}_{h}}}=\mathcal{N} \cdot \boldsymbol{\psi}=0 .
$$

Next, we will show the equivalence of brackets $\{\cdot, \cdot\}_{1}$ and $\{\cdot, \cdot\}_{2}$. After integration by parts of (3.16), we obtain

$$
\begin{aligned}
\{\mathcal{F}, \mathcal{H}\}_{1}= & \sum_{K \in \mathcal{T}_{h}} \int_{K}\left(\frac{f_{h}}{B_{h}} \frac{\delta F^{\perp}}{\delta \boldsymbol{v}_{h}} \cdot \frac{\delta H}{\delta \boldsymbol{v}_{h}}\right) \mathrm{d} \Omega \\
& +\sum_{K \in \mathcal{T}_{h}} \int_{K}\left(\mathcal{D} \cdot\left(\frac{\delta F}{\delta \boldsymbol{v}_{h}}\right) \frac{\delta H}{\delta \eta_{h}}+\left(\mathcal{D} \frac{\delta F}{\delta \eta_{h}}\right) \cdot \frac{\delta H}{\delta \boldsymbol{v}_{h}}\right) \mathrm{d} \Omega \\
& +\sum_{K \in \mathcal{T}_{h}} \int_{\partial K}\left(\widehat{\mathcal{N} \cdot \frac{\delta F}{\delta \boldsymbol{v}_{h}}} \frac{\delta H}{\delta \eta_{h}}-\widehat{\mathcal{N} \cdot \frac{\delta H}{\delta \boldsymbol{v}_{h}}} \frac{\delta F}{\delta \eta_{h}}-\mathcal{N} \cdot \frac{\delta F}{\delta \boldsymbol{v}_{h}} \frac{\delta H}{\delta \eta_{h}}\right) \mathrm{d} S,
\end{aligned}
$$

where we have used that the functional derivatives of $F$ on an element $K \in \mathcal{T}_{h}$ are equal to the arbitrary test functions $\psi$ and $\phi$, which are zero outside each element $K$. Therefore it is not necessary to introduce a numerical flux on the last contribution in the integral over the element boundary in (3.20). We introduce now the numerical fluxes (3.17) and boundary conditions (3.18)-(3.19) and use that each interior edge occurs twice in the summation over all elements in the tessellation. The integral over the element boundaries in (3.20) can then be expressed as

$$
\begin{gathered}
\sum_{K \in \mathcal{T}_{h}} \int_{\partial K}\left(\widehat{\mathcal{N} \cdot \frac{\delta F}{\delta \boldsymbol{v}_{h}}} \frac{\delta H}{\delta \eta_{h}}-\widehat{\mathcal{N} \cdot \frac{\delta H}{\delta \boldsymbol{v}_{h}}} \frac{\delta F}{\delta \eta_{h}}-\mathcal{N} \cdot \frac{\delta F}{\delta \boldsymbol{v}_{h}} \frac{\delta H}{\delta \eta_{h}}\right) \mathrm{d} S \\
=\sum_{e \in \Gamma_{i}} \int_{e}\left(\mathcal{N}_{L} \cdot\left((1-\theta) \frac{\delta F}{\delta \boldsymbol{v}_{h}^{L}}+\theta \frac{\delta F}{\delta \boldsymbol{v}_{h}^{R}}\right)\left(\frac{\delta H}{\delta \eta_{h}^{L}}-\frac{\delta H}{\delta \eta_{h}^{R}}\right)\right.
\end{gathered}
$$




$$
\begin{aligned}
& +\mathcal{N}_{L} \cdot\left((1-\theta) \frac{\delta H}{\delta \boldsymbol{v}_{h}^{L}}+\theta \frac{\delta H}{\delta \boldsymbol{v}_{h}^{R}}\right)\left(\frac{\delta F}{\delta \eta_{h}^{R}}-\frac{\delta F}{\delta \eta_{h}^{L}}\right) \\
& \left.+\mathcal{N}_{L} \cdot \frac{\delta F}{\delta \boldsymbol{v}_{h}^{R}} \frac{\delta H}{\delta \eta_{h}^{R}}-\mathcal{N}_{L} \cdot \frac{\delta F}{\delta \boldsymbol{v}_{h}^{L}} \frac{\delta H}{\delta \eta_{h}^{L}}\right) \mathrm{d} S,
\end{aligned}
$$

which can be simplified into

$$
\begin{aligned}
\sum_{K \in \mathcal{T}_{h}} & \int_{\partial K}\left(\widehat{\mathcal{N} \cdot \frac{\delta F}{\delta \boldsymbol{v}_{h}}} \frac{\delta H}{\delta \eta_{h}}-\widehat{\mathcal{N} \cdot \frac{\delta H}{\delta \boldsymbol{v}_{h}}} \frac{\delta F}{\delta \eta_{h}}-\mathcal{N} \cdot \frac{\delta F}{\delta \boldsymbol{v}_{h}} \frac{\delta H}{\delta \eta_{h}}\right) \mathrm{d} S \\
= & \sum_{e \in \Gamma_{i}} \int_{e}\left(\mathcal{N}_{L} \cdot\left(\frac{\delta F}{\delta \boldsymbol{v}_{h}^{R}}-\frac{\delta F}{\delta \boldsymbol{v}_{h}^{L}}\right)\left(\theta \frac{\delta H}{\delta \eta_{h}^{L}}+(1-\theta) \frac{\delta H}{\delta \eta_{h}^{R}}\right)\right. \\
& \left.+\mathcal{N}_{L} \cdot\left((1-\theta) \frac{\delta H}{\delta \boldsymbol{v}_{h}^{L}}+\theta \frac{\delta H}{\delta \boldsymbol{v}_{h}^{R}}\right)\left(\frac{\delta F}{\delta \eta_{h}^{R}}-\frac{\delta F}{\delta \eta_{h}^{L}}\right)\right) \mathrm{d} S .
\end{aligned}
$$

Combining (3.20) and (3.21) the final result equals (3.15) and proves that the bracket $\{\cdot, \cdot\}_{2}$ is also skew-symmetric.

The skew-symmetry and the form of the discrete bracket immediately implies the following properties:

Proposition 3.1 (Energy and mass conservation) The solution to the Hamiltonian formulation (3.10)-(3.11) satisfies energy and mass conservation at the discrete level, i.e.

$$
\frac{\mathrm{d}}{\mathrm{d} t} H=0 \quad \text { and } \quad \frac{\mathrm{d}}{\mathrm{d} t} M=0,
$$

where

$$
H=\frac{1}{2} \sum_{K} \int_{K}\left(B_{h}\left|\boldsymbol{v}_{h}\right|^{2}+C_{h} \eta_{h}^{2}\right) \mathrm{d} \Omega \quad \text { and } \quad M=\sum_{K} \int_{K} \eta_{h} \mathrm{~d} \Omega .
$$

\subsection{DG Scheme}

In this section, we compare the discontinuous Hamiltonian formulation with a DG formulation. Multiplying (2.1) with arbitrary test functions $\psi \in V_{h} \times V_{h}$ and $\phi \in V_{h}$, and integrating by parts over each element $K \in \mathcal{T}_{h}$, we obtain the following relation for $\boldsymbol{v}_{h} \in V_{h} \times V_{h}$ and $\eta_{h} \in V_{h}$ :

$$
\begin{aligned}
& \int_{K} \frac{\partial \boldsymbol{v}_{h}}{\partial t} \cdot \boldsymbol{\psi} \mathrm{d} \Omega=\int_{K}\left(-f \boldsymbol{v}_{h}^{\perp} \cdot \boldsymbol{\psi}+C_{h} \eta_{h} \mathcal{D} \cdot \boldsymbol{\psi}\right) \mathrm{d} \Omega-\int_{\partial K} \widehat{C_{h} \eta_{h}} \mathcal{N} \cdot \boldsymbol{\psi} \mathrm{d} s, \\
& \left.\int_{K} \frac{\partial \eta_{h}}{\partial t} \phi \mathrm{d} x \mathrm{~d} y=\int_{K}\left(B_{h} \boldsymbol{v}_{h}\right) \cdot \mathcal{D} \phi \mathrm{d} \Omega-\int_{\partial K} \mathcal{N} \widehat{\cdot\left(B_{h} \boldsymbol{v}_{h}\right.}\right) \phi \mathrm{d} S,
\end{aligned}
$$

where we choose, motivated by the numerical fluxes used for the Hamiltonian formulation, discussed in Sects. 3.3-3.4, and stability reasons [14], the following alternating numerical fluxes

$$
\begin{aligned}
& \widehat{C_{h} \eta_{h}}=\theta C_{h} \eta_{h}^{L}+(1-\theta) C_{h} \eta_{h}^{R}, \\
& \left.\widehat{\mathcal{N} \cdot\left(B_{h} \boldsymbol{v}_{h}\right.}\right)=(1-\theta) \mathcal{N} \cdot\left(B_{h} \boldsymbol{v}_{h}\right)^{L}+\theta \mathcal{N} \cdot\left(B_{h} \boldsymbol{v}_{h}\right)^{R}, \quad 0 \leq \theta \leq 1 .
\end{aligned}
$$


At edges at the domain boundary we impose the physical boundary condition (2.2), which states

$$
\mathcal{N} \cdot \boldsymbol{v}_{h}=0 .
$$

The DG scheme (3.22)-(3.23) for constant $B_{h}$ and $C_{h}$ equals the Hamiltonian discontinuous finite element scheme and then also satisfies energy conservation. These restrictions imply that $B_{h} \boldsymbol{v}_{h}$ and $C_{h} \eta_{h}$ belong to the Galerkin test function space. For general nonconstant $B_{h}$ and $C_{h}$, energy conservation can not be obtained from the classical DG method (3.22)(3.24). In the linear case, however, we can weight the usual test function with $B_{h}$ to alleviate this problem.

\section{Time Discretization}

We compare two time discretization methods. First, the non-symplectic and dissipative thirdorder total variation diminishing Runge-Kutta method of [13] is used. Next, we consider a symplectic splitting method for Hamiltonian systems [6].

\subsection{Third Order TVD Runge-Kutta}

An explicit third order Runge-Kutta method [13] is used for solving

$$
\dot{u}=L(u, t),
$$

where the spatial discretization operator $L(u, t)$ is defined as

$$
\begin{aligned}
u^{(1)} & =u^{n}+\Delta t L\left(u^{n}, t^{n}\right), \\
u^{(2)} & =\frac{3}{4} u^{n}+\frac{1}{4} u^{(1)}+\frac{1}{4} \Delta t L\left(u^{(1)}, t^{n}+\Delta t\right), \\
u^{n+1} & =\frac{1}{3} u^{n}+\frac{2}{3} u^{(2)}+\frac{2}{3} \Delta t L\left(u^{(2)}, t^{n}+\frac{1}{2} \Delta t\right) .
\end{aligned}
$$

\subsection{Symplectic Splitting Method}

The TVD Runge-Kutta method discussed in Sect. 4.1 is slightly dissipative. We consider a symplectic time integration method to ensure phase space conservation, and avoid energy loss [5]. In symplectic schemes the energy conservation is generally approximated as the numerical approximation of the energy tends to oscillate around a mean value. A splitting scheme is used in time such that in each splitting step the scheme is solved exactly and conserved. The following ordinary differential equations arise from the Hamiltonian spatial discretization for each element

$$
\begin{aligned}
M_{i j} \frac{\mathrm{d} \hat{\boldsymbol{v}}_{j}}{\mathrm{~d} t} & =-O_{i j} \hat{\mathbf{Q}}_{j}+\mathbf{G}_{i j} \hat{r}_{j}-\int_{\partial K} \mathcal{N} \hat{r}_{h} \psi_{i} \mathrm{~d} s, \\
M_{i j} \frac{\mathrm{d} \hat{\eta}_{j}}{\mathrm{~d} t} & =\mathbf{G}_{i j} \cdot \hat{\mathbf{Q}}_{j}-\int_{\partial K} \widehat{\mathcal{N} \cdot \mathbf{Q}_{h}} \psi_{i} \mathrm{~d} s \\
M_{i j} \hat{\mathbf{Q}}_{j} & =B_{i j} \hat{\boldsymbol{v}}_{j} \quad \text { and } \quad M_{i j} \hat{r}_{j}=C_{i j} \hat{\eta}_{j}
\end{aligned}
$$


with elemental coefficients $\hat{r}_{j}$ and $\hat{\boldsymbol{v}}_{j}$, basis functions $\psi_{i}$ or $\psi_{j}$, and elemental matrices

$$
\begin{aligned}
M_{i j} & =\int_{K} \psi_{i} \psi_{j} \mathrm{~d} x \mathrm{~d} y, \\
B_{i j} & =\int_{K} B_{h} \psi_{i} \psi_{j} \mathrm{~d} x \mathrm{~d} y, \\
C_{i j} & =\int_{K} C_{h} \psi_{i} \psi_{j} \mathrm{~d} x \mathrm{~d} y, \\
\mathbf{G}_{i j} & =\int_{K} \psi_{j} \mathcal{D} \psi_{i} \mathrm{~d} x \mathrm{~d} y, \\
O_{i j} & =\int_{K} \frac{f_{h}}{B_{h}} \psi_{i} \psi_{j} \mathrm{~d} x \mathrm{~d} y .
\end{aligned}
$$

We write the Hamiltonian spatial discretization (4.3)-(4.4) abstractly in the form

$$
\frac{\mathrm{d} \hat{\boldsymbol{v}}_{K}}{\mathrm{~d} t}=L_{1} \hat{\boldsymbol{v}}_{K}+L_{2} \hat{\eta}, \quad \frac{\mathrm{d} \hat{\eta}_{K}}{\mathrm{~d} t}=L_{3} \hat{\boldsymbol{v}},
$$

where $\hat{\boldsymbol{v}}$ and $\hat{\eta}$ are the expansion coefficients, specifically denoted in element $K$ by $\hat{\boldsymbol{v}}_{K}$ and $\hat{\eta}_{K}$, and $L_{1}, L_{2}, L_{3}$ are the appropriate constant matrices independent of the variables. The matrix $L_{1}$ only involves local integrals per element and no face integrals, as follows from (4.3). In contrast, $L_{2}$ and $L_{3}$ depend on face integrals as well. Note that, for conciseness of presentation, we explicitly eliminated $\mathbf{Q}_{h}$ and $r_{h}$.

The linear system (4.5) is a generalized Poisson system with a quadratic Hamiltonian depending on a quadratic term with velocity coefficients $\hat{v}$ plus one with coefficients $\hat{\eta}$. A second order symplectic partitioned Runge-Kutta time integration method is now based on this splitting of the Hamiltonian $H=H_{v}+H_{\eta}$ in two separate quadratic parts. The resulting time discretization consists of the composition of exactly integrable pieces, one half step of the Hamiltonian dynamics using only the Hamiltonian $H_{v}$, a complete time step using the Hamiltonian $H_{\eta}$, and then one half time step using only the Hamiltonian $H_{v}$ again. We therefore employ exactly integrable linear Poisson systems in turn, as suggested in [6]. For $f=0$ we then basically obtain the Störmer-Verlet method for our linear system [6]. For nonzero and constant $f$, and constant function $B$, the velocity $\hat{v}$ in the first and last split time step can be solved exactly. For nonzero and nonconstant $f$ and $B$, the solution for $\hat{\boldsymbol{v}}$ in a split time step is local and harmonic, but its coefficients require a numerical determination. The resulting symplectic method does require a constant time step. The linear dispersion relations would yield approximate time step requirements.

With $\tau=\Delta t$, and the case with $f$ and $B$ constant, the resulting numerical scheme for (4.5) becomes

$$
\begin{aligned}
& \tilde{\mathbf{U}}_{i n t}=\frac{1}{f}\left(\begin{array}{cc}
\sin (f \tau / 2) & (1-\cos (f \tau / 2)) \\
(\cos (f \tau / 2)-1) & \sin (f \tau / 2)
\end{array}\right)\left(\begin{array}{c}
\hat{u}^{n} \\
\hat{v}^{n}
\end{array}\right), \\
& \hat{\eta}^{n+1 / 2}=\hat{\eta}^{n}+L_{3} \tilde{\mathbf{U}}_{i n t}, \\
& \forall K:\left(\begin{array}{c}
\hat{u}_{j}^{n+1 / 2} \\
\hat{v}_{j}^{n+1 / 2}
\end{array}\right)=\left(\begin{array}{cc}
\cos (f \tau / 2) & \sin (f \tau / 2) \\
-\sin (f \tau / 2) & \cos (f \tau / 2)
\end{array}\right)\left(\begin{array}{c}
\hat{u}^{n} \\
\hat{v}^{n}
\end{array}\right), \\
& \tilde{\boldsymbol{v}}^{n+1}=\hat{\boldsymbol{v}}^{n+1 / 2}-\tau L_{2} \hat{\eta}^{n+1 / 2},
\end{aligned}
$$




$$
\begin{aligned}
& \forall K:\left(\begin{array}{l}
\hat{u}_{j}^{n+1} \\
\hat{v}_{j}^{n+1}
\end{array}\right)=\left(\begin{array}{cc}
\cos (f \tau / 2) & \sin (f \tau / 2) \\
-\sin (f \tau / 2) & \cos (f \tau / 2)
\end{array}\right)\left(\begin{array}{c}
\tilde{u}^{n+1} \\
\tilde{v}^{n+1}
\end{array}\right), \\
& \mathbf{U}_{i n t}^{n+1}=\frac{1}{f}\left(\begin{array}{cc}
\sin (f \tau / 2) & (1-\cos (f \tau / 2)) \\
(\cos (f \tau / 2)-1) & \sin (f \tau / 2)
\end{array}\right)\left(\begin{array}{c}
\hat{u}^{n+1} \\
\hat{v}^{n+1}
\end{array}\right), \\
& \hat{\eta}^{n+1}=\hat{\eta}^{n+1 / 2}+L_{3} \mathbf{U}_{i n t}^{n+1} .
\end{aligned}
$$

In (4.6a) with both $f$ and $B$ constant, we have obtained $\mathbf{U}_{\text {int }}$ by integrating the following ordinary differential equations for the local coefficients $\hat{\boldsymbol{v}}_{j}$ per element exactly:

$$
\frac{\mathrm{d} \hat{\boldsymbol{v}}_{j}}{\mathrm{~d} t}=-f \hat{\boldsymbol{v}}_{j}^{\perp} .
$$

For nonconstant $f$ and $B$, the splitting scheme in time becomes slightly more involved.

\section{Numerical Results}

In this section, we provide numerical examples to illustrate the accuracy and capability of the methods developed in the previous section. In all examples, the figures present the solution obtained with a particular choice of the mesh. We have verified that the results shown are numerically convergent with the aid of successive mesh refinements, in all cases.

\subsection{Rotating Linear Shallow Water Equations}

In the first set of test cases we consider wave problems governed by the rotating linear shallow water equations on an $f$-plane, these are given by (2.11). The tests involve harmonic waves, Kelvin and Poincaré waves, and linear waves in a closed parabolic bowl.

\subsubsection{Harmonic Waves}

Consider a two-dimensional (2D) harmonic wave solution of (2.11) for constant topography $D=H$ and constant $f$ in a periodic domain $L_{x} \times L_{y}$. Exact solutions of this problem are given in Appendix A. First, we consider the accuracy for the parameters given in (A.2). The $L^{2}$ and $L^{\infty}$ errors and the numerical orders of accuracy for the water depth $\eta$ are given in Table 1 at time $t=1$ on a uniform rectangular mesh in a domain $[0,1] \times[0,1]$. We see that the method with $P^{k}$ elements gives a uniform $(k+1)$-th order of accuracy in both norms.

We also present the wave profile using $P^{1}$ elements on a uniform rectangular $80 \times 80$ mesh at $t=100$ for the parameters given in (A.3). In Fig. 1, the water depth $\eta$ is shown at time $t=100$ as well as the energy as function of time. The discontinuous Hamiltonian and DG formulation coincide in this case, and we therefore compare the Runge-Kutta and symplectic splitting time discretizations. The results show that the symplectic time integration scheme is better in energy conservation than a third order TVD Runge-Kutta method.

\subsubsection{Kelvin and Poincaré Waves}

We consider Kelvin and Poincaré waves for the shallow water equations (2.11). These are specific normal-mode solutions of the rotating shallow water equations. Kelvin waves arise as boundary-trapped modes in the presence of rotation; on the Northern hemisphere where $f>0$ these modes propagate counterclockwise with the boundary on the right. Poincaré 
Table 1 Accuracy test for the water depth $\eta$ of the linear shallow water equations (2.11) with exact solution (A.1). Periodic boundary conditions. Uniform meshes containing $N_{x} \times N_{y}$ cells at time $t=1$

\begin{tabular}{llllll}
\hline & $N_{x} \times N_{y}$ & $L^{2}$ error & Order & $L^{\infty}$ error & Order \\
\hline$P^{0}$ & $20 \times 20$ & $3.70 \mathrm{E}-01$ & - & $1.13 \mathrm{E}-00$ & - \\
& $40 \times 40$ & $1.48 \mathrm{E}-01$ & 1.32 & $4.54 \mathrm{E}-01$ & 1.31 \\
& $80 \times 80$ & $8.89 \mathrm{E}-02$ & 0.74 & $2.87 \mathrm{E}-01$ & 0.66 \\
& $160 \times 160$ & $5.01 \mathrm{E}-02$ & 0.83 & $1.58 \mathrm{E}-01$ & 0.86 \\
$P^{1}$ & $20 \times 20$ & $8.86 \mathrm{E}-02$ & - & $3.94 \mathrm{E}-01$ & - \\
& $40 \times 40$ & $1.75 \mathrm{E}-02$ & 2.34 & $9.36 \mathrm{E}-02$ & 2.07 \\
& $80 \times 80$ & $5.11 \mathrm{E}-03$ & 1.78 & $2.28 \mathrm{E}-02$ & 2.04 \\
& $160 \times 160$ & $1.10 \mathrm{E}-03$ & 2.22 & $5.17 \mathrm{E}-03$ & 2.14 \\
$P^{2}$ & $20 \times 20$ & $2.09 \mathrm{E}-02$ & - & $9.61 \mathrm{E}-02$ & - \\
& $40 \times 40$ & $1.67 \mathrm{E}-03$ & 3.64 & $7.49 \mathrm{E}-03$ & 3.68 \\
& $80 \times 80$ & $1.95 \mathrm{E}-04$ & 3.09 & $1.38 \mathrm{E}-03$ & 2.44 \\
& $160 \times 160$ & $1.93 \mathrm{E}-05$ & 3.34 & $7.61 \mathrm{E}-05$ & 4.18 \\
$P^{3}$ & $20 \times 20$ & $1.84 \mathrm{E}-03$ & - & $1.17 \mathrm{E}-02$ & - \\
& $40 \times 40$ & $1.22 \mathrm{E}-04$ & 3.92 & $6.06 \mathrm{E}-04$ & 4.27 \\
& $80 \times 80$ & $6.68 \mathrm{E}-06$ & 4.19 & $4.10 \mathrm{E}-05$ & 3.88 \\
& $160 \times 160$ & $3.85 \mathrm{E}-07$ & 4.11 & $2.26 \mathrm{E}-06$ & 4.18 \\
\hline \multirow{3}{*}{40} & & & & &
\end{tabular}
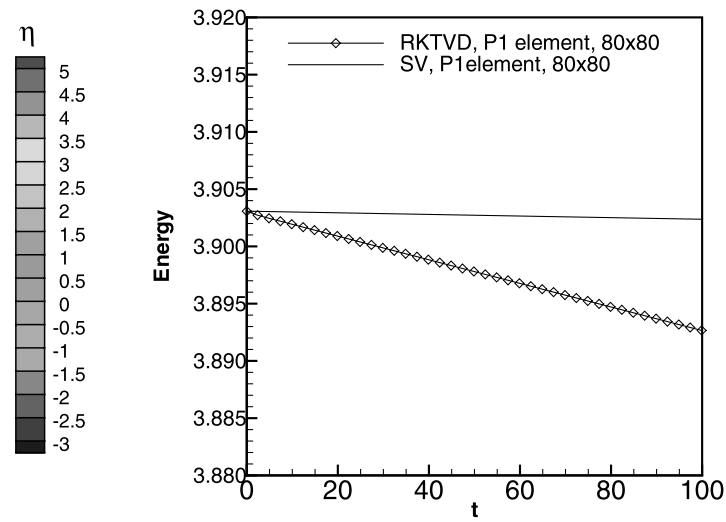

Fig. 1 Harmonic waves described by the linear rotating shallow water equations (2.11) at $t=100$ and the discrete energy for the symplectic splitting (SV) and TVD Runge-Kutta (RKTVD) time integration methods

modes are gravity modes modified by the Earth's rotation. These eigenmodes in turn test the numerical scheme in the presence of boundaries and rotation.

The exact solutions for three different cases are given in Appendices B.1, B.2 and B.3. In Figs. 2 and 3, we show respectively Kelvin waves and Poincaré waves at $t=100$ in a rectangular channel periodic in $x$ using $P^{1}$ elements on an unstructured triangular mesh (1000 elements). We also plot the discrete energy using the TVD Runge-Kutta (TVDRK) and the symplectic splitting (SV) time integration methods. In Fig. 4, we show the Poincaré waves in a circular basin using $P^{1}$ elements on an unstructured triangular mesh (1000 elements) after 100 periods and the discrete energy for the symplectic splitting time integration schemes. The TVD Runge-Kutta method, however, did not survive a long time simulation 

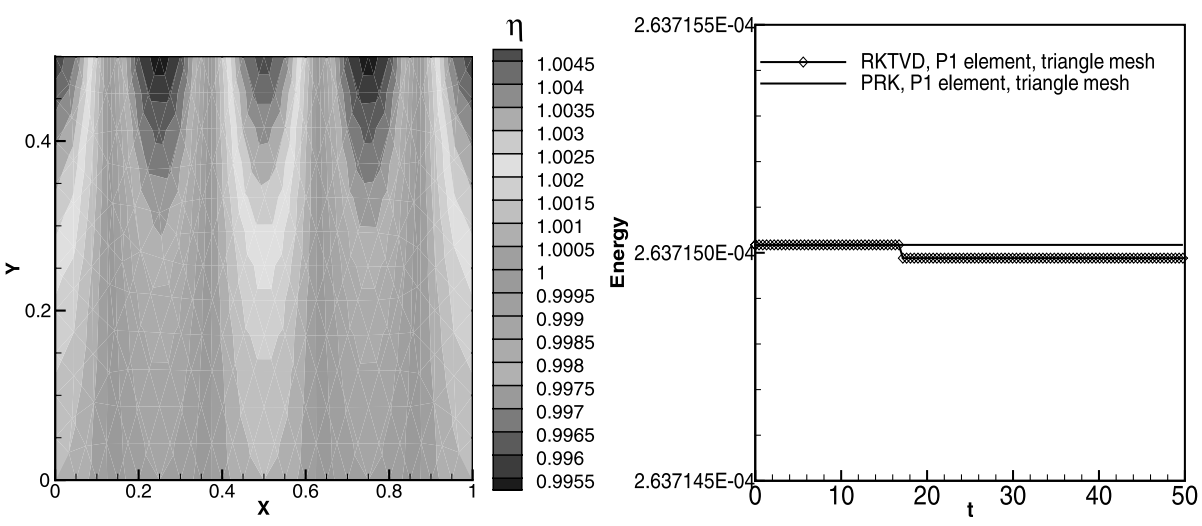

Fig. 2 Kelvin waves described by the linear rotating shallow water equations (2.11) in a rectangular domain after 100 periods and the discrete energy for the TVD Runge-Kutta (TVDRK) and the symplectic splitting (PRK) time integration methods
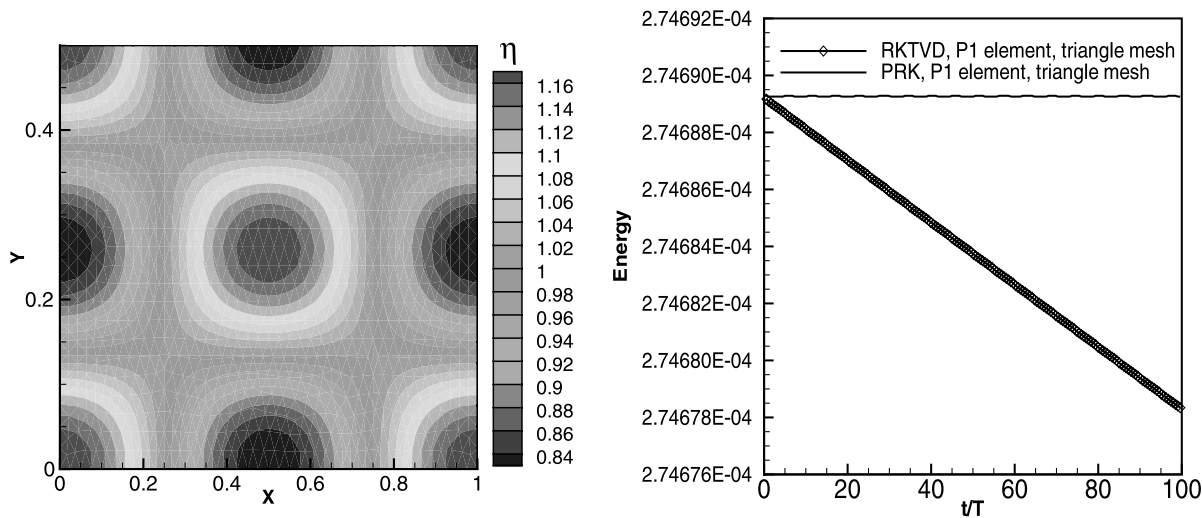

Fig. 3 Poincaré waves described by the linear rotating shallow water equations (2.11) in a rectangular domain after 100 periods and the discrete energy for the TVD Runge-Kutta (TVDRK) and the symplectic splitting (PRK) time integration methods

for the Poincaré waves and will blowup after a few wave periods. We therefore only give the energy results for the symplectic scheme.

The Hamiltonian and the DG finite element scheme coincide in these test cases because the bottom topography is constant. The energy in all these examples is conserved very well with the discontinuous Hamiltonian discretization in combination with the splitting time integration method, even for unstructured meshes and with solid wall boundary conditions. The results show that the symplectic scheme is more accurate in conserving energy and also more stable than a third order TVD Runge-Kutta method.

\subsubsection{Linear Waves in Closed Parabolic Bowl}

To test the Hamiltonian discretization against the classical non-Hamiltonian DG scheme, we consider linear non-rotating shallow water (2.11) with $f=0$ in a closed circular parabolic bowl. Hence, the topography is varying: $D=D(x, y)=D_{0}\left(1-\left(x^{2}+y^{2}\right) / a^{2}\right)$. One 

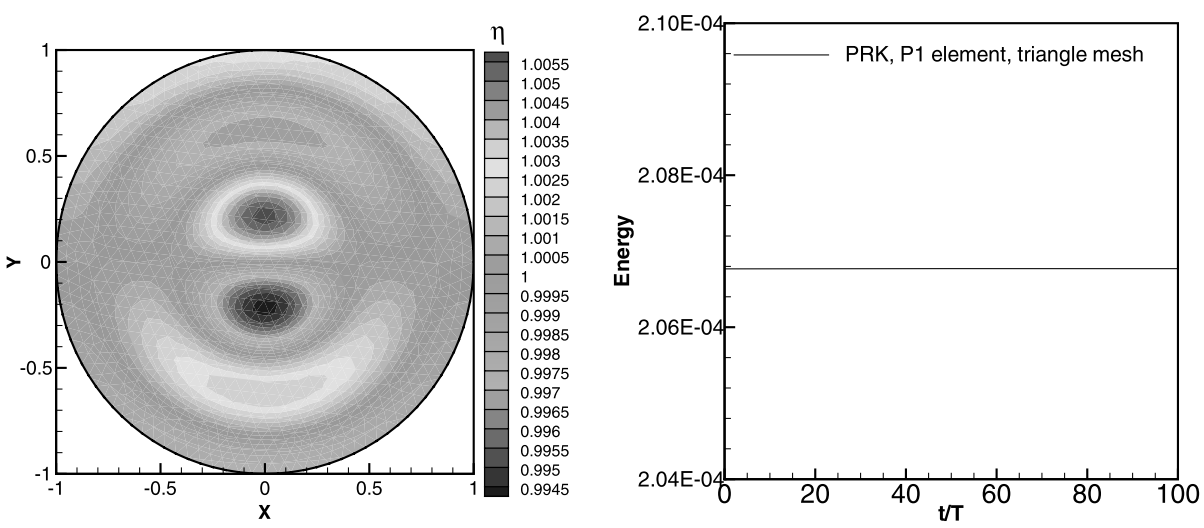

Fig. 4 Poincaré waves described by the linear rotating shallow water (2.11) in a circular domain after 100 periods and the discrete energy for the symplectic splitting (PRK) time integration methods. The TVDRK method is unstable for this case
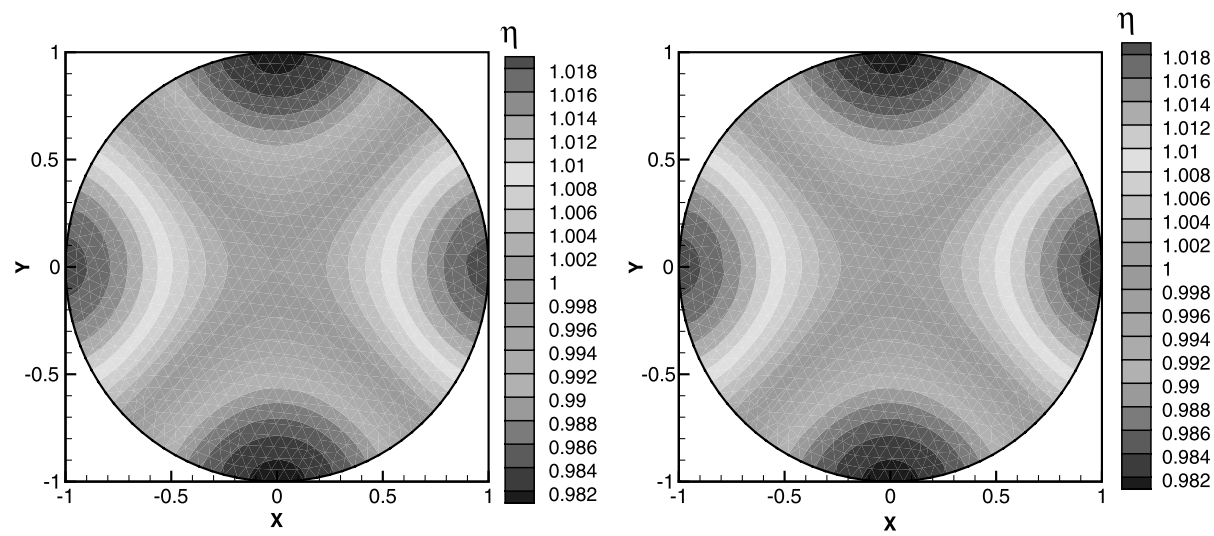

Fig. 5 Coastal shelf waves for equation described by the linear rotating shallow water equations (2.11) after 100 periods. Left: Hamiltonian discretization. Right: DG scheme, for $s=2$

of the exact solutions of (2.11) is given in Appendix C. In Fig. 5, we show the waves by the Hamiltonian formulation and the DG scheme in a circular basin for $P^{1}$ elements with unstructured triangular meshes (1000 elements) for (2.11) with the parameter $s=2$ after 100 periods. The result in Fig. 6 shows that the discontinuous Hamiltonian discretization and the DG scheme can both approximately preserve the discrete energy for very high spatial resolution. In Fig. 7, however, we also give the discrete energy for equations (2.11) when the resolution is lower, for the parameter value $s=30$. In this result, the difference between the discontinuous Hamiltonian discretization and the DG scheme becomes obvious. The discontinuous Hamiltonian discretization performs very well regarding conservation of the discrete energy. The discrete energy of the DG scheme is oscillatory for the SV scheme and growing for the TVDRK method. 
Fig. 6 The energy is shown for an eigenmode in a circular basin over 100 periods for the Hamiltonian and DG spatial discretizations, and both Runge-Kutta and Störmer-Verlet time discretizations, for $s=2$

Fig. 7 The energy is shown for an eigenmode in a circular basin over 100 periods for the Hamiltonian and DG spatial discretizations, and both Runge-Kutta and Störmer-Verlet time discretizations, for $s=30$
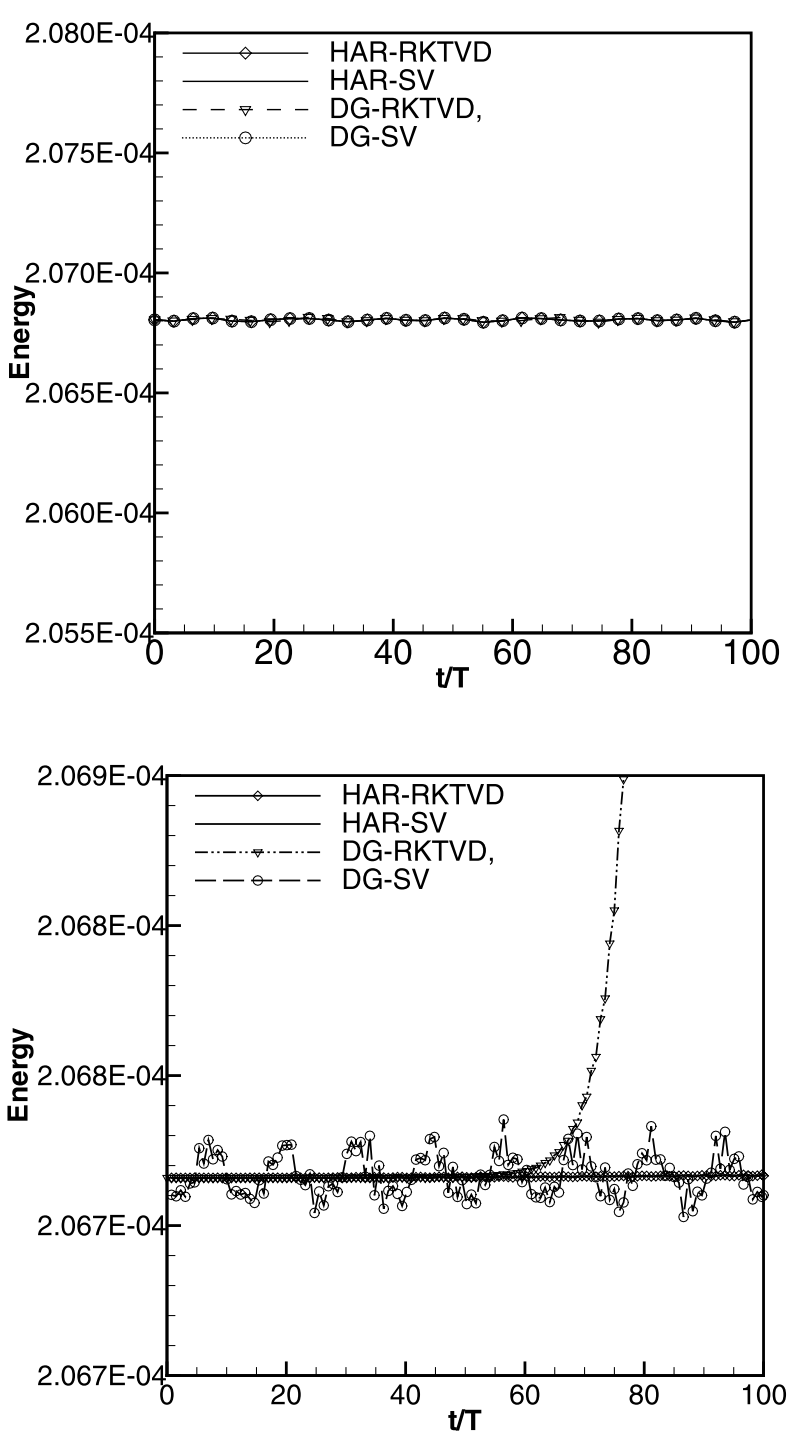

\subsection{Two-Dimensional Maxwell Equations}

Consider the two-dimensional Maxwell equations

$$
\frac{\partial \boldsymbol{H}}{\partial t}=\nabla^{\perp} E_{z}, \quad \frac{\partial E_{z}}{\partial t}=\nabla^{\perp} \boldsymbol{H},
$$

with uniform dielectric permittivity and magnetic permeability. The computational domain is $[0,2 \pi / \alpha] \times[0,2 \pi / \beta]$ with periodic boundary conditions. The final simulation time is $t=100$ (100 periods). The smooth and non-smooth exact solutions of (5.1) used in this test case are given in Appendix D. 
Table 2 Errors in the $x$-component of the magnetic field $H_{x}$ for smooth solution (D.1) of the Maxwell equation (5.1) at $t=100$

\begin{tabular}{llllll}
\hline & $N_{x} \times N_{y}$ & $L^{2}$ error & Order & $L^{\infty}$ error & Order \\
\hline$P^{0}$ & $20 \times 20$ & $4.29 \mathrm{E}-01$ & - & $1.02 \mathrm{E}-00$ & - \\
& $40 \times 40$ & $1.81 \mathrm{E}-01$ & 1.24 & $5.24 \mathrm{E}-01$ & 0.96 \\
& $80 \times 80$ & $5.88 \mathrm{E}-02$ & 1.62 & $1.94 \mathrm{E}-01$ & 1.43 \\
& $160 \times 160$ & $2.09 \mathrm{E}-02$ & 1.49 & $8.17 \mathrm{E}-02$ & 1.25 \\
$P^{1}$ & $20 \times 20$ & $3.74 \mathrm{E}-02$ & - & $1.92 \mathrm{E}-01$ & - \\
& $40 \times 40$ & $4.64 \mathrm{E}-03$ & 3.01 & $3.96 \mathrm{E}-02$ & 2.28 \\
& $80 \times 80$ & $9.98 \mathrm{E}-04$ & 2.22 & $9.20 \mathrm{E}-03$ & 2.10 \\
& $160 \times 160$ & $2.47 \mathrm{E}-04$ & 2.01 & $2.28 \mathrm{E}-03$ & 2.02 \\
$P^{2}$ & $20 \times 20$ & $2.09 \mathrm{E}-03$ & - & $1.75 \mathrm{E}-02$ & - \\
& $40 \times 40$ & $2.26 \mathrm{E}-04$ & 3.21 & $2.22 \mathrm{E}-03$ & 2.98 \\
& $80 \times 80$ & $2.82 \mathrm{E}-05$ & 3.00 & $3.01 \mathrm{E}-04$ & 2.88 \\
& $160 \times 160$ & $3.47 \mathrm{E}-06$ & 3.02 & $3.60 \mathrm{E}-05$ & 3.06 \\
\hline
\end{tabular}

\begin{tabular}{llllll}
\hline & $N_{x} \times N_{y}$ & $L^{2}$ error & Order & $L^{\infty}$ error & Order \\
\hline$P^{0}$ & $20 \times 20$ & $3.12 \mathrm{E}-01$ & - & $7.42 \mathrm{E}-01$ & - \\
& $40 \times 40$ & $1.32 \mathrm{E}-01$ & 1.24 & $3.81 \mathrm{E}-01$ & 0.96 \\
& $80 \times 80$ & $4.28 \mathrm{E}-02$ & 1.62 & $1.41 \mathrm{E}-01$ & 1.43 \\
& $160 \times 160$ & $1.52 \mathrm{E}-02$ & 1.49 & $5.93 \mathrm{E}-02$ & 1.25 \\
$P^{1}$ & $20 \times 20$ & $2.78 \mathrm{E}-02$ & - & $1.45 \mathrm{E}-01$ & - \\
& $40 \times 40$ & $3.41 \mathrm{E}-03$ & 3.03 & $2.93 \mathrm{E}-02$ & 2.30 \\
& $80 \times 80$ & $7.27 \mathrm{E}-04$ & 2.23 & $6.72 \mathrm{E}-03$ & 2.13 \\
& $160 \times 160$ & $1.80 \mathrm{E}-04$ & 2.01 & $1.66 \mathrm{E}-03$ & 2.02 \\
$P^{2}$ & $20 \times 20$ & $1.56 \mathrm{E}-03$ & - & $1.43 \mathrm{E}-02$ & - \\
& $40 \times 40$ & $1.70 \mathrm{E}-04$ & 3.45 & $2.50 \mathrm{E}-03$ & 3.12 \\
& $80 \times 80$ & $2.08 \mathrm{E}-05$ & 3.03 & $2.29 \mathrm{E}-04$ & 2.83 \\
& $160 \times 160$ & $2.60 \mathrm{E}-06$ & 3.00 & $2.77 \mathrm{E}-05$ & 3.04 \\
\hline
\end{tabular}

Table 3 Errors in the $y$-component of the magnetic field $H_{y}$ for smooth solution (D.1) of the Maxwell equation (5.1) at $t=100$
For the smooth solution, the $L^{2}$ and $L^{\infty}$ errors and the numerical orders of accuracy for $H_{x}, H_{y}$ and $E_{z}$ on a uniform rectangular mesh using periodic boundary conditions are presented in Tables 2, 3 and 4. For this constant coefficient case the Hamiltonian and DG schemes are identical.

We can see that the discretization using $P^{k}$ elements gives a uniform $(k+1)$-th order of accuracy in both norms. We also show the discrete energy in Fig. 8 using $P^{1}$ elements on a uniform rectangular $80 \times 80$ mesh. The results show that the symplectic scheme is better than a third order TVD Runge-Kutta method in energy conservation.

For the non-smooth solution we show the numerical results using $P^{1}$ elements on a uniform rectangular $80 \times 80$ mesh for $H_{x}, H_{y}$ and $E_{z}$ of solution (D.2a) at $t=100$ in Fig. 9 . The energy shown in Fig. 10 is also conserved very well for the singular solution using the symplectic time integration scheme, whereas the third order TVD Runge-Kutta method is dissipative. 
Table 4 Errors in the $z$-component of the electric field $E_{z}$ for smooth solution (D.1) of the Maxwell equation (5.1) at $t=100$

Fig. 8 Energy for smooth solution (D.1) of the Maxwell equation (5.1)

\begin{tabular}{llllll}
\hline & $N_{x} \times N_{y}$ & $L^{2}$ error & Order & $L^{\infty}$ error & Order \\
\hline$P^{0}$ & $20 \times 20$ & $4.76 \mathrm{E}-01$ & - & $1.25 \mathrm{E}-00$ & - \\
& $40 \times 40$ & $1.64 \mathrm{E}-01$ & 1.53 & $5.75 \mathrm{E}-01$ & 1.12 \\
& $80 \times 80$ & $4.82 \mathrm{E}-02$ & 1.77 & $2.09 \mathrm{E}-01$ & 1.46 \\
& $160 \times 160$ & $1.77 \mathrm{E}-02$ & 1.44 & $7.53 \mathrm{E}-02$ & 1.47 \\
$P^{1}$ & $20 \times 20$ & $4.26 \mathrm{E}-02$ & - & $1.57 \mathrm{E}-01$ & - \\
& $40 \times 40$ & $5.18 \mathrm{E}-03$ & 3.04 & $4.31 \mathrm{E}-02$ & 1.86 \\
& $80 \times 80$ & $1.14 \mathrm{E}-03$ & 2.18 & $1.11 \mathrm{E}-02$ & 1.95 \\
& $160 \times 160$ & $2.82 \mathrm{E}-04$ & 2.02 & $2.80 \mathrm{E}-03$ & 2.00 \\
$P^{2}$ & $20 \times 20$ & $2.10 \mathrm{E}-03$ & - & $2.18 \mathrm{E}-02$ & - \\
& $40 \times 40$ & $1.92 \mathrm{E}-04$ & 3.45 & $2.50 \mathrm{E}-03$ & 3.12 \\
& $80 \times 80$ & $2.37 \mathrm{E}-05$ & 3.02 & $3.09 \mathrm{E}-04$ & 3.02 \\
& $160 \times 160$ & $2.99 \mathrm{E}-06$ & 3.00 & $4.11 \mathrm{E}-05$ & 2.91 \\
\hline
\end{tabular}

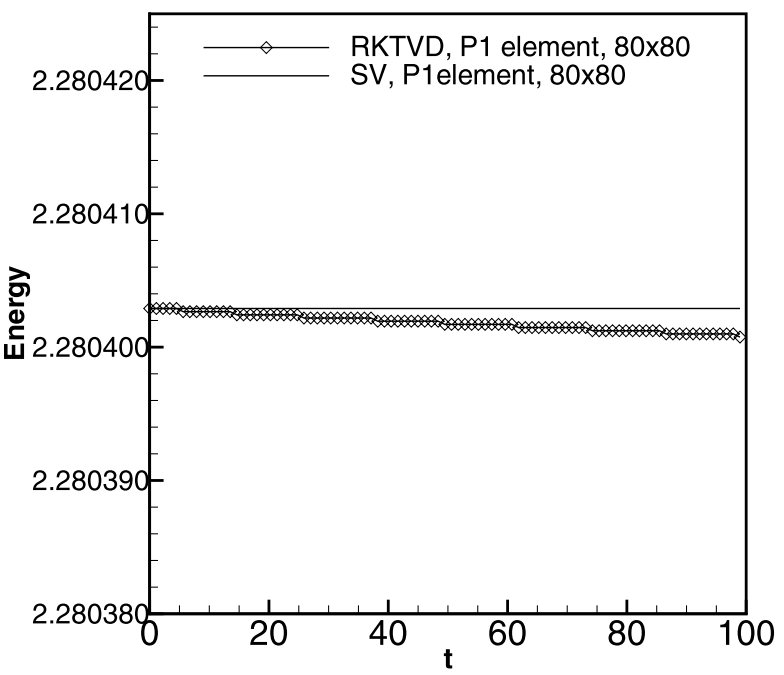

\section{Conclusion}

In this article we have developed a discontinuous finite element discretization for Hamiltonian systems of linear hyperbolic equations, which conserves energy and phase-space structure because it preserves the skew-symmetry of the Poisson bracket at the discrete level. For comparison, we also have presented a classical DG method. Numerical examples illustrate the accuracy and capability of the method. These examples show that the discontinuous Hamiltonian finite element discretization developed in this article in combination with a symplectic splitting method for the time integration preserves the discrete energy approximately but without drift, even on unstructured meshes. This makes the discontinuous Hamiltonian discretization an excellent numerical scheme for long time integration, e.g., in physical problems.

In contrast, the discontinuous Galerkin discretization only preserves the discrete energy in the constant coefficient case, but not in general. A crucial part of the Hamiltonian method 

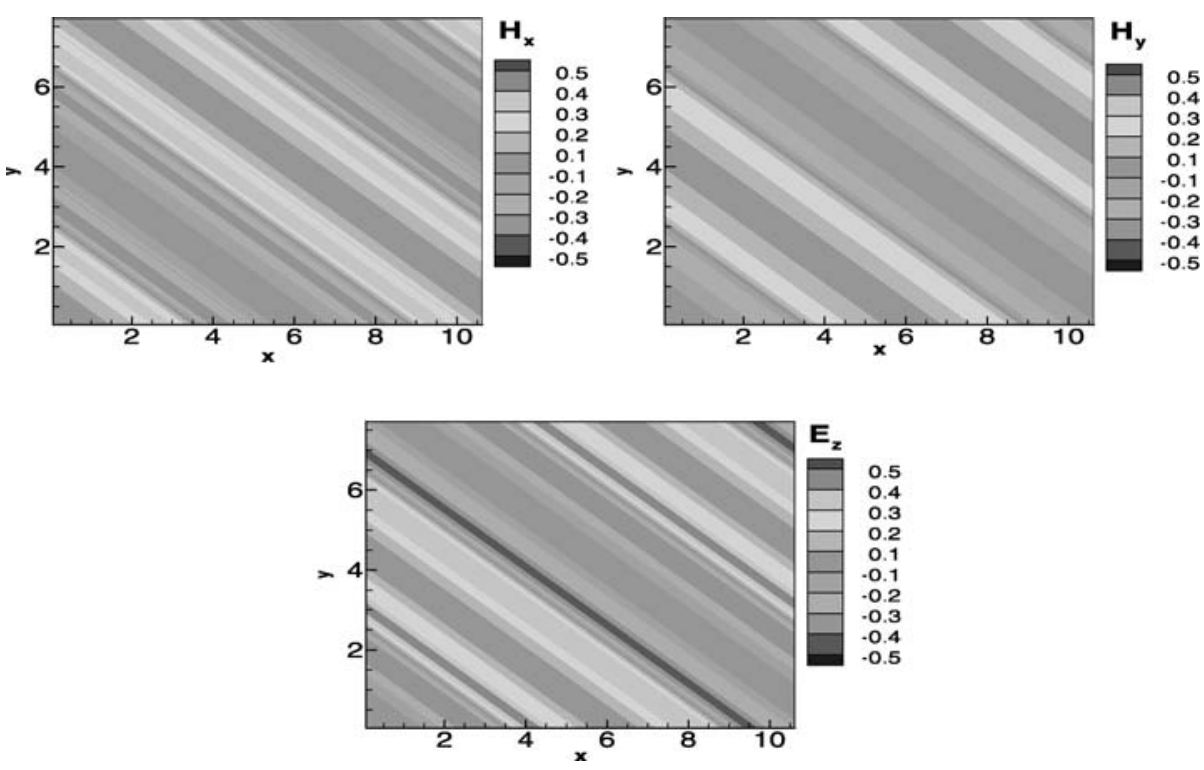

Fig. 9 The contour plots for $H_{x}, H_{y}$ and $E_{z}$ of solution (D.2a) at $t=100$

Fig. 10 Energy for non-smooth solution (D.2a) of the Maxwell equations (5.1)

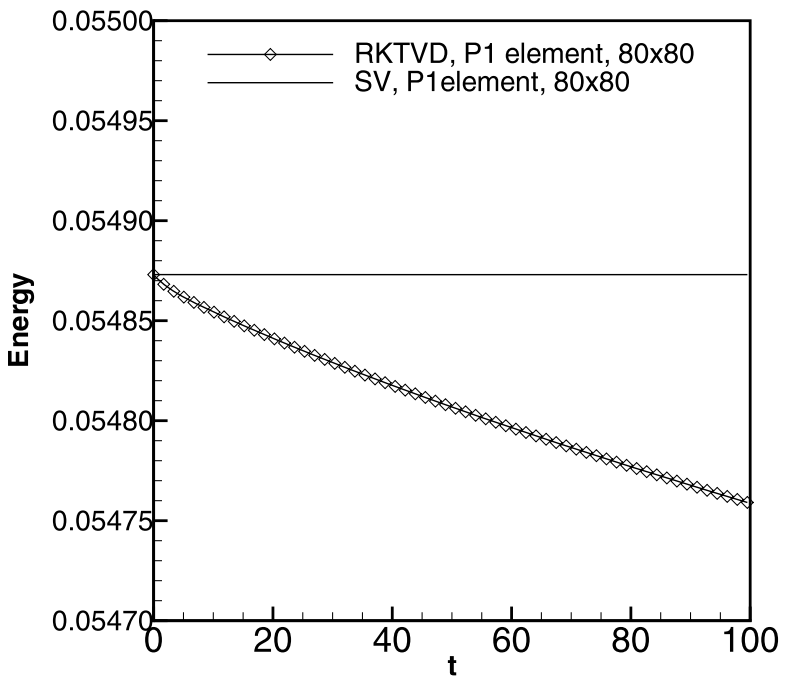

is the $L^{2}$-projection of the Hamiltonian functional derivatives on the Galerkin test functions. As an alternative time integration method, we also considered the simpler, third order accurate TVD Runge-Kutta time integration. This time integration method results, however, in most test cases in a decrease of the discrete energy. Although not addressed in this article, the methodology is expected to apply to other cases, such as the generalized linear system of Huttunen et al. [7] and the three-dimensional acoustic equations [2]. We plan to explore these applications in our future research. 
Acknowledgements O.B. was supported by a fellowship of the Netherlands Academy of Arts and Sciences during part of this research. Research of Y.X. was supported by NSFC grant 10601055 and "A Foundation for the Author of Excellent Doctoral Dissertation of the Chinese Academy of Sciences". We thank Albert Wildeman, Bob Peeters and Sid Visser for valuable discussions on the symplectic time splitting method.

Open Access This article is distributed under the terms of the Creative Commons Attribution Noncommercial License which permits any noncommercial use, distribution, and reproduction in any medium, provided the original author(s) and source are credited.

\section{Appendix A: Exact Harmonic Solution of (2.11)}

The exact solution of (2.11) with periodic boundary conditions on a domain $L_{x} \times L_{y}$ is:

$$
\begin{aligned}
& \eta=\sum_{s= \pm 1} \sum_{m, n=-\infty}^{\infty}\left(A_{m n s} \cos (z)+B_{m n s} \sin (z)\right), \\
& u=\sum_{s= \pm 1} \sum_{m, n=-\infty}^{\infty}\left(C_{m n s} \cos (z)+D_{m n s} \sin (z)\right), \\
& v=\sum_{s= \pm 1} \sum_{m, n=-\infty}^{\infty}\left(E_{m n s} \cos (z)+F_{m n s} \sin (z)\right),
\end{aligned}
$$

with

$$
z=k_{m} x+l_{n} y+\omega_{m n s} t
$$

where

$$
\begin{aligned}
C_{m n s} & =g \frac{k_{m} \omega_{m n s} A_{m n s}-f l_{n} B_{m n s}}{f^{2}-\omega_{m n s}^{2}}, \\
D_{m n s} & =g \frac{k_{m} \omega_{m n s} B_{m n s}+f l_{n} A_{m n s}}{f^{2}-\omega_{m n s}^{2}}, \\
E_{m n s} & =g \frac{l_{n} \omega_{m n s} A_{m n s}+f k_{m} B_{m n s}}{f^{2}-\omega_{m n s}^{2}}, \\
F_{m n s} & =g \frac{l_{m} \omega_{m n s} B_{m n s}-f k_{m} A_{m n s}}{f^{2}-\omega_{m n s}^{2}}, \\
k_{m} & =\frac{2 \pi m}{L_{x}}, \quad l_{n}=\frac{2 \pi n}{L_{y}}, \\
\omega_{m n \pm} & = \pm \sqrt{\left(f^{2}+g H\left(k_{m}^{2}+l_{n}^{2}\right)\right)}
\end{aligned}
$$

with $m, n$ are integers. The coefficients $A_{m n s}$ and $B_{m n s}$ are arbitrary amplitudes with indices $m, n, s$; and, $L_{x}$ and $L_{y}$ are the lengths of the domain in the $x$ and $y$ directions, respectively. We have used the following two sets of parameters in our numerical tests

$$
\begin{aligned}
& f=1, \quad g=1, \quad H=1, \\
& k_{1}=1, \quad l_{1}=1, \quad s_{1}=1, \quad A_{1}=1, \quad B_{1}=1 \\
& k_{2}=2, \quad l_{2}=-3, \quad s_{2}=-1, \quad A_{2}=0.8, \quad B_{2}=0.6 .
\end{aligned}
$$


and

$$
\begin{array}{ll}
f=1, & g=1, \quad H=1, \\
k_{1}=1, & l_{1}=1, \quad s_{1}=1, \quad A_{1}=1, \quad B_{1}=1 \\
k_{2}=2, & l_{2}=-3, \quad s_{2}=-1, \quad A_{2}=0.8, \quad B_{2}=0.6, \\
k_{3}=4, & l_{3}=5, \quad s_{3}=1, \quad A_{3}=1.2, \quad B_{3}=1.5 .
\end{array}
$$

\section{Appendix B: Kelvin and Poincaré Waves}

\section{B.1 Kelvin Wave in a Rectangular Domain}

A Kelvin wave in a rectangular domain $\left[0, L_{x}\right] \times\left[0, L_{y}\right]$ is given by

$$
\begin{aligned}
& u(x, y, t)=\frac{(\omega k-f l) g A}{f^{2}-\omega^{2}} e^{l y} \cos (k x+\omega t), \\
& v(x, y, t)=0, \\
& \eta(x, y, t)=H+A e^{l y} \cos (k x+\omega t),
\end{aligned}
$$

with $a^{2}=g H$, periodic boundary conditions in the $x$ direction and solid wall boundary conditions in the $y$ direction. $H$ is the mean free surface height, $\omega=a k$ is the dispersion relation, $l=f / a, k=2 \pi m / L_{x}$ are the wave numbers, and $m$ is an arbitrary integer. The parameters used are the following: $A=0.001, H=1.0, L_{x}=1.0, L_{y}=0.5, m=2, g=1$, $f=3.193379349$.

\section{B.2 Poincaré Wave in a Rectangular Domain}

A Poincaré wave in a rectangular domain $\left[0, L_{x}\right] \times\left[0, L_{y}\right]$ is given by

$$
\begin{aligned}
& u(x, y, t)=\frac{g A}{f^{2}-\omega^{2}}\left(-k l\left(f^{2}-\omega^{2}\right) \cos (l y)+f k \sin (l y)\right) \cos (k x+\omega t), \\
& v(x, y, t)=-\frac{g A}{f^{2}-\omega^{2}}\left((f k)^{2}+(\omega l)^{2}\right) \sin (l y) \sin (k x+\omega t), \\
& \eta(x, y, t)=H+A(\omega l \cos (l y)+f k \sin (l y)) \cos (k x+\omega t),
\end{aligned}
$$

with $a^{2}=g H$, periodic boundary conditions in $x$ and solid wall boundary conditions in $y$. $\omega^{2}=f^{2}+a^{2}\left(k^{2}+l^{2}\right)$ is the dispersion relation, $k=2 \pi m / L_{x}, l=2 \pi n / L_{y}$ are the wave numbers, and $m, n$ are integers. The parameters used are the following: $A=1.0 E-0.5$, $H=1.0, L_{x}=1.0, L_{y}=0.5, m=1, n=1, g=1, f=3.193379349$.

\section{B.3 Poincaré Wave in a Circular Basin}

In polar coordinates with $r$ the radius and $\theta$ the azimuthal angle, the Poincare wave in a circular basin of radius $R$ is given by

$$
\begin{aligned}
& u_{r}(r, \theta, t)=\frac{g A}{f^{2}-\omega^{2}}\left(-\frac{m}{r}(f+\omega) \mathrm{F}_{m}(k r)+\omega k \mathrm{~F}_{m+1}(k r)\right) \cos (m \theta+\omega t), \\
& u_{\theta}(r, \theta, t)=\frac{g A}{f^{2}-\omega^{2}}\left(\frac{\omega m}{r}(f+\omega) \mathrm{F}_{m}(k r)-f k \omega k \mathrm{~F}_{m+1}(k r)\right) \sin (m \theta+\omega t), \\
& \eta(r, \theta, t)=H+A \mathrm{~F}_{m}(k r) \sin (m \theta+\omega t)
\end{aligned}
$$


with $a^{2}=g H$, and solid wall boundary conditions at $r=R . \mathrm{F}_{m}(z)=J_{m}(z)$ are Bessel functions of the first kind, $\omega^{2}=f^{2}+a^{2} k^{2}$ is the dispersion relation, and the wave number $k$ has to satisfy the following relations due to the solid wall boundary conditions at $r=R$ :

$$
f m \mathrm{~F}_{m}(k R)+w k \mathrm{~F}_{m+1}(k R)=0 .
$$

The parameters used are the following: $A=0.01, H=1.0, R=1, k=8.55806886, m=1$, $n=1, g=1, f=1.596689674$.

\section{Appendix C: Linear Waves in Closed Parabolic Bowl}

From the solution in [8] (Sect. 193), we take the following case with $\alpha=n=s+4, \mathrm{i}^{2}=-1$ and in our notation $\zeta=\eta$, to obtain the following solution in polar coordinates

$$
\begin{aligned}
& \eta(r, \theta, t)=A_{s}\left(\frac{r}{a}\right)^{s}\left(1-\frac{(s+2)}{(s+1)}\left(\frac{r}{a}\right)^{2}\right) e^{\mathrm{i}(\sigma t+s \theta)} \\
& u_{r}(r, \theta, t)=\frac{g \mathrm{i}}{\sigma a} A_{s}\left(\frac{r}{a}\right)^{s-1}\left(s-\frac{(s+2)^{2}}{(s+1)}\left(\frac{r}{a}\right)^{2}\right) e^{\mathrm{i}(\sigma t+s \theta)} \\
& u_{\theta}(r, \theta, t)=-\frac{g s}{\sigma r} A_{s}\left(\frac{r}{a}\right)^{s}\left(1-\frac{(s+2)}{(s+1)}\left(\frac{r}{a}\right)^{2}\right) e^{\mathrm{i}(\sigma t+s \theta)}
\end{aligned}
$$

with $s$ a positive integer and

$$
R=a \sqrt{\frac{s(s+1)}{(s+2)^{2}}}<a,
$$

as required for positivity of $D(r)$, and to satisfy the slip boundary condition $u(R, t) \propto$ $\left.\partial_{r} \eta\right|_{r=R}=0$. Furthermore, the rest depth is:

$$
D(r)=D_{0}\left(1-r^{2} / a^{2}\right)
$$

The real part of (C.1a) gives one of the desired modes

$$
\begin{aligned}
& \eta(r, \theta, t)=A_{s}\left(\frac{r}{a}\right)^{s}\left(1-\frac{(s+2)}{(s+1)}\left(\frac{r}{a}\right)^{2}\right) \cos (\sigma t+s \theta), \\
& u_{r}(r, \theta, t)=-\frac{g}{\sigma a} A_{s}\left(\frac{r}{a}\right)^{s-1}\left(s-\frac{(s+2)^{2}}{(s+1)}\left(\frac{r}{a}\right)^{2}\right) \sin (\sigma t+s \theta), \\
& u_{\theta}(r, \theta, t)=-\frac{g s}{\sigma r} A_{s}\left(\frac{r}{a}\right)^{s}\left(1-\frac{(s+2)}{(s+1)}\left(\frac{r}{a}\right)^{2}\right) \cos (\sigma t+s \theta) .
\end{aligned}
$$

The frequency for the case $\alpha=n=s+4$ is given by

$$
\sigma^{2}=g D_{0}(6 s+8) / a^{2}
$$

The parameter values used are: $s=2$, or $30, A_{s}=0.1, D_{0}=1, R=1, g=1, a=$ $R \sqrt{(s+2)^{2} /[s(s+1)]}$. 


\section{Appendix D: Exact Solutions of the Maxwell Equations}

A smooth exact solution of the Maxwell equation (5.1) is

$$
\left(\begin{array}{l}
H_{x} \\
H_{y} \\
E_{z}
\end{array}\right)=\left(\begin{array}{c}
-\beta \\
\alpha \\
1
\end{array}\right) \exp (\cos (\alpha x+\beta y+t)) .
$$

A solution for (5.1) with a singularity is

$$
\begin{aligned}
\left(\begin{array}{l}
H_{x} \\
H_{y} \\
E_{z}
\end{array}\right) & =\left(\begin{array}{c}
-\beta \\
\alpha \\
1
\end{array}\right) \varphi((\cos (\alpha x+\beta y+t))), \\
\varphi(w) & = \begin{cases}w \log |w|, & \text { if } w \neq 0, \\
0, & \text { if } w=0,\end{cases}
\end{aligned}
$$

where $\alpha=\cos (0.3 \pi)$ and $\beta=\sin (0.3 \pi)$.

\section{References}

1. Bokhove, O., Oliver, M.: Parcel Eulerian-Lagrangian fluid dynamics for rotating geophysical flows. Proc. R. Soc. A. 462, 2563-2573 (2006)

2. Blom, C.: Discontinuous Galerkin method on tetrahedral elements for aeroacoustic. Ph.D. Thesis, University of Twente, Enschede, The Netherlands (2003)

3. Cockburn, B., Hou, S., Shu, C.-W.: The Runge-Kutta local projection discontinuous Galerkin finite element method for conservation laws IV: the multidimensional case. Math. Comput. 54, 545-581 (1990)

4. Cockburn, B., Shu, C.-W.: The Runge-Kutta discontinuous Galerkin method for conservation laws V: multidimensional systems. J. Comput. Phys. 141, 199-224 (1998)

5. Hairer, E., Lubich, C., Wanner, G.: Geometric Numerical Integration: Structure Preserving Algorithms for Ordinary Differential Equations. Springer, Heidelberg (2002)

6. Hairer, E., Lubich, C., Wanner, G.: Geometric numerical integration illustrated by the Störmer-Verlet method. Acta Numerica 12, 399-450 (2003)

7. Huttunen, T., Monk, P., Collino, F., Kaipio, J.P.: The ultra-weak variational formulation for elastic wave problems. SIAM J. Sci. Comput. 25, 1717-1742 (2004)

8. Lamb, H.: Hydrodynamics, 6th edn. Cambridge University Press, London (1975)

9. Lighthill, J.: Waves in Fluids. Cambridge University Press, London (1978)

10. Marsden, J.E., Ratiu, T.S.: Introduction to mechanics and symmetry: a basic exposition of classical mechanical systems. In: Texts in Applied Mathematics, vol. 17. Springer, New York (1994)

11. Morrison, P.J.: Hamiltonian description of the ideal fluid. Rev. Mod. Phys. 70, 467-521 (1998)

12. Salmon, R.: Lectures on Geophysical Fluid Dynamics. Oxford University Press, Oxford (1998)

13. Shu, C.-W., Osher, S.: Efficient implementation of essentially non-oscillatory shock-capturing schemes. J. Comput. Phys. 77, 439-471 (1988)

14. Yan, J., Shu, C.-W.: Local discontinuous Galerkin methods for partial differential equations with higher order derivatives. J. Sci. Comput. 17, 27-47 (2002) 